\title{
Estimating the movements of terrestrial animal populations using broad-scale occurrence data
}

\author{
Sarah R. Supp ${ }^{1 *} \mathbb{D}$, Gil Bohrer ${ }^{2}$, John Fieberg ${ }^{3}$ and Frank A. La Sorte ${ }^{4}$
}

\begin{abstract}
As human and automated sensor networks collect increasingly massive volumes of animal observations, new opportunities have arisen to use these data to infer or track species movements. Sources of broad scale occurrence datasets include crowdsourced databases, such as eBird and iNaturalist, weather surveillance radars, and passive automated sensors including acoustic monitoring units and camera trap networks. Such data resources represent static observations, typically at the species level, at a given location. Nonetheless, by combining multiple observations across many locations and times it is possible to infer spatially continuous population-level movements. Population-level movement characterizes the aggregated movement of individuals comprising a population, such as range contractions, expansions, climate tracking, or migration, that can result from physical, behavioral, or demographic processes. A desire to model population movements from such forms of occurrence data has led to an evolving field that has created new analytical and statistical approaches that can account for spatial and temporal sampling bias in the observations. The insights generated from the growth of population-level movement research can complement the insights from focal tracking studies, and elucidate mechanisms driving changes in population distributions at potentially larger spatial and temporal scales. This review will summarize current broad-scale occurrence datasets, discuss the latest approaches for utilizing them in population-level movement analyses, and highlight studies where such analyses have provided ecological insights. We outline the conceptual approaches and common methodological steps to infer movements from spatially distributed occurrence data that currently exist for terrestrial animals, though similar approaches may be applicable to plants, freshwater, or marine organisms.
\end{abstract}

Keywords: Acoustic monitoring, Camera trap, Crowdsourced data, eBird, Migration, Occurrence data, Populationlevel movement, Range expansion, Terrestrial animals, Weather surveillance radar

\section{Background}

Describing how the locations of individuals or populations change across space and through time is an important part of understanding many different levels of ecological organization. Tracked movements can allow us to understand individual behaviors (e.g., establishing home range, mate seeking, emigration to new territory

\footnotetext{
*Correspondence: supps@denison.edu

${ }^{1}$ Data Analytics Program, Denison University, Granville, $\mathrm{OH} 43023$, USA

Full list of author information is available at the end of the article
}

[1-3]), the consequences of inter- or intra-specific interactions (e.g., competitive, facilitative $[4,5]$ ), how individuals track and acquire resources (e.g., follow resource pulses, seasonality, migration [6-8]), or the effects of natural or anthropogenic perturbations (e.g., relocating from catastrophe or land use change, shifting habitat use in response to climate change or changes in resource availability $[9,10])$. Recent technological innovations have expanded our ability to document fine-scale movements, and to track individuals over both short and long distances and time periods. Tracking sensors are becoming original author(s) and the source, provide a link to the Creative Commons licence, and indicate if changes were made. The images or other third party material in this article are included in the article's Creative Commons licence, unless indicated otherwise in a credit line to the material. If material is not included in the article's Creative Commons licence and your intended use is not permitted by statutory regulation or exceeds the permitted use, you will need to obtain permission directly from the copyright holder. To view a copy of this licence, visit http://creativecommons.org/licenses/by/4.0/. The Creative Commons Public Domain Dedication waiver (http://creativeco mmons.org/publicdomain/zero/1.0/) applies to the data made available in this article, unless otherwise stated in a credit line to the data. 
smaller, more affordable, and are being applied to an expanding range of taxa (e.g., birds, whales, bats, insects, fish $[11,12])$. From such devices, the movements of individuals can be tracked, allowing researchers to connect known locations through time to understand how and where individuals move, or to evaluate the movements of many individuals to understand interactive components of movements among conspecifics or between species, or to summarize how aggregate populations move through time (e.g., $[13,14])$.

Tracking data, however, are often limited to a small number of individuals $(<30)$ over short time periods (days to months), restricting the ability of researchers to generate broad-scale inferences [15-17]. In addition, individual-level tracking data are often constrained due to organisms or species having small body size $[18,19]$, budgetary limitations [20], or high tag loss (anatomical, behavioral, animal safety [21-23]). It can also be challenging to mark and track a sample of individuals that adequately represent broadly distributed species or species with large populations (e.g., [24, 25]). Because of these limitations, it is often more feasible to collect individual locations where identity is not retained over time (occurrence data), especially across large spatial and temporal scales. Even where individual-level movement data exist, complementary approaches that instead use individual occurrence data to study emergent dynamics in population distributions, referred to as populationlevel movement (previously described in [26-28]), have the potential to address knowledge gaps and advance our understanding of general movement phenomena (Fig. 1), ecological interactions, disease spread [29], invasive species and range-expanding species [30-32], climate response [7, 10, 14, 33, 34], and conservation of mobile populations, such as those that migrate [35].

Population-level movement can be summarized by an aggregate metric of the population distribution (e.g., its center or boundary) and quantified by its rate of change (direction, magnitude) across a subset of individuals within a defined population, species, or geographic area. Population-level movement, or redistribution, includes migration, nomadism, and the shifts of previously sedentary ranges or established natal or breeding dispersal areas [26, 37, 38]. Movement at the population-level can result from individual behavior, demographic processes, external factors, or their combined effects (Fig. 2). Despite the strengths of individual-level tracking data, researchers are increasingly able to turn towards occurrence data from human and automated sensor networks to infer macro-scale population-level movements [11, 39, 40]. Recent advancements in data acquisition, processing, and analysis have allowed broad-scale occurrence datasets to be used to infer spatially continuous

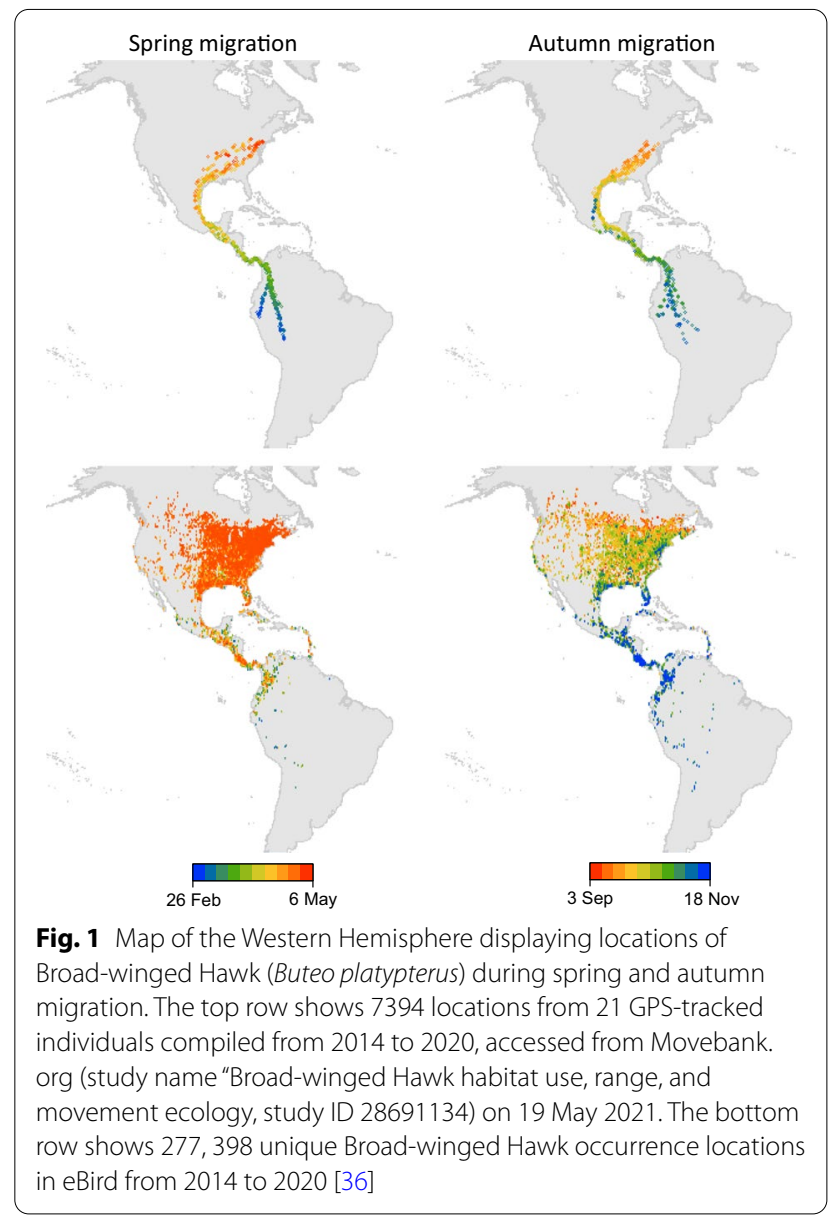

movement of populations across the landscape over short to long timescales. Adding a macroecological lens to movement ecology provides a novel perspective for connecting individual processes and behaviors to emergent population-level movements across a range of temporal scales (seasonal, interannual, multi-generational, or evolutionary) and the big-picture trends in geographic range movement, expansion, or contraction that are occurring in response to ongoing regional and global changes [41].

Animal occurrence data, often collected as static presence, presence/absence, counts, or density in space and time along with a measure of sampling effort, are increasingly available through widely distributed sensor networks. These sensors can be human, as in the case of crowdsourced initiatives where tasks such as data collection or processing are outsourced to an undefined and large group of volunteers [43]. In other cases, automated sensor networks collect data passively. Examples include weather surveillance radar [39], acoustic monitoring [44], or image monitoring $[12,45]$. Some efforts overlap, with automated sensors passively collecting data, through a crowdsourced network of volunteer maintainers (e.g., 


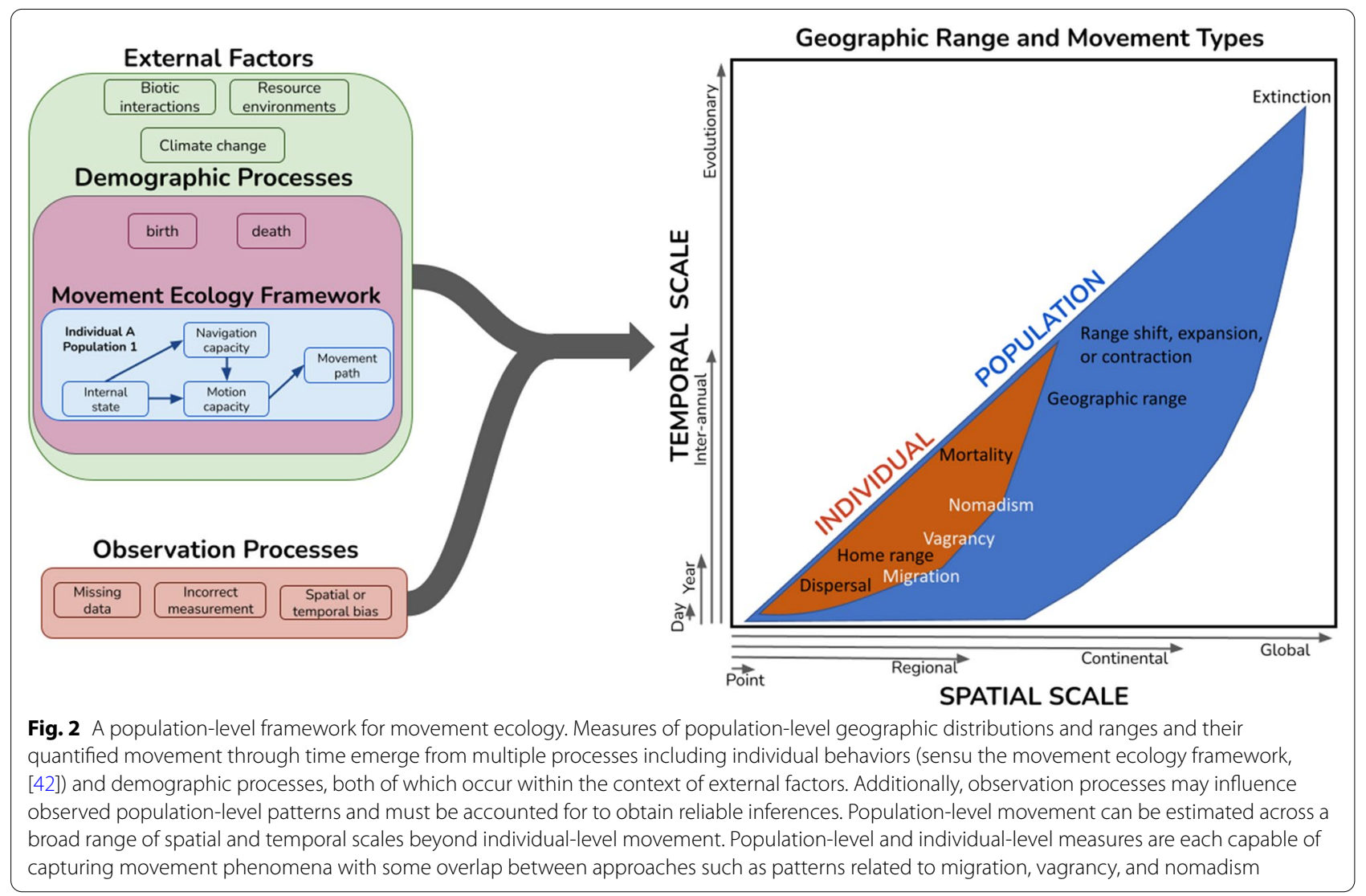

$[46,47])$. In all cases, each occurrence record represents an individual observation, a count, or a density of individuals, but in most cases, records lack individual identifiers that would allow linking the same individual animal to multiple locations at different times, and thus, explicitly record movement. To ascertain movement patterns, general requirements are that the network of sensors be distributed across a broad spatial extent, and that the sensor network collects data at a high enough temporal frequency relative to the movement properties of the species in order to detect shifts in location, and relative to the needs of the research question. For example, to infer population-level movement of a seasonally migrating species, a sensor network would need to include observations from across the summer and winter ranges and within regions of passage, with occurrences documented frequently throughout the species' annual life cycle (Fig. 1).

Many datasets provide publicly available occurrence location data (Table 1), and the past decade has seen an increase in the number of datasets and new methods developed to infer population movement from occurrence data. Such occurrence datasets are commonly used to estimate species distributions at a single point in time or by pooling data across years (e.g., [48, 49]), which generally represent a static perspective of species location or occupancy across a landscape. Species distribution models are also often used to forecast changes in distribution in response to changes in climate or land use (e.g., [50, 51]). While species distribution models do not directly estimate movement, they can be used to measure different aspects of a population's location in time and space, and by linking changes in those measures across time, one can infer patterns in populationlevel movement that include migration, nomadism, or range shifts. To date, little synthesis has been done on the data, methods, and types of models used to infer population-level movement from occurrence data. The purpose of this review is to (1) to lay out a theoretical framework to understand and guide population-level movement research, (2) summarize existing broad-scale species occurrence datasets, (3) discuss the latest statistical and modeling approaches for inferring populationlevel movement, and (4) highlight where such studies have provided ecological insights. Through the review, we hope to provide guidance to researchers conducting their own movement analysis using occurrence data, and to identify areas ripe for future research or development. We restrict our discussion to population-level movements in terrestrial animals, mostly at regional and larger 
spatial scales, and seasonal or longer temporal scales, though similar insights may be applicable to marine or aquatic animal studies, or at finer spatial or temporal resolutions (Box 1).

\section{A theoretical framework for population-level movement}

Recent statistical and modeling advances have allowed researchers to use occurrence data to infer spatially continuous population-level movement across the landscape. The types of population-level movement questions that can be answered with occurrence data differ from traditional individual-level movement questions using tracking data. Occurrence data are well suited for documenting movements between regions that occur in a relatively short time frame, such as days, weeks or months (migration, nomadism, or dispersal; sensu [17, $37,38]$ ) or for documenting movements and geographic range shifts that occur across a relatively long time frame, such as multiple years, generations, or evolutionary time $[55,56]$. In contrast, occurrence data are poorly suited for determining the movements within resident populations (e.g., encamped movements related to behavioral activities like foraging). Beyond describing a movement path, the movement ecology paradigm identified three basic components focused on individual movement-internal state, navigation capacity, motion capacity-and their correlation with external factors [42]. Importantly, since occurrence data frequently represent locations where individual identity is not retained over time, movement paths cannot be described for specific individuals, and variation in movement cannot be attributed to individual characteristics such as age, genetics, phenotype, behavior, or interactions [57]. Instead, occurrence data can be used to identify population-level movement patterns that emerge from demographic processes and the movement of individuals. Population distributions can be compared within or across years or between different groups (i.e., species or regions), and changes or differences in distribution may be used to discern external abiotic or biotic correlates of movement.

We outline a population-level movement framework that builds upon the individual-level movement ecology paradigm [42], recognizing that population-level distributions emerge from individual movement mechanisms [26] and movement types [38] along with demographic processes (Fig. 2). This framework recognizes the disparate spatial and temporal scales at which the individual- and population-level processes often play out and organizes different movement types within these scales. Emergent changes in population distribution (i.e., population-level movement) may be influenced

Box 1 Key terminology needed to use this review as a guide

\section{Glossary}

Tracking data Locations of uniquely identified individuals that are linked through time

Occurrence data Locations that cannot be identified to specific individuals but can be labeled as belonging to a particular population, species, or taxonomic group. Occurrence records at a location may be measured as presence, count, or density values

Population The group of individual observations to which one wants to make inference. For example, a subset of individuals within the same or multiple species, a subpopulation within a defined geographic area, a meta-population considered as a whole across a region, or even a whole species spread across a region or continent. This definition differs somewhat from a biological population, and is more similar to a statistical population, which is defined by the set of observations of interest for a specific question

Individual-level movement A movement path generated by linking locations of the same individual through time

Population-level movement Population redistribution over time, which can be summarized by an aggregate metric such as center or boundary, and quantified by its rate of change in direction or magnitude. Movement at the population level can result from individual behavior, demographic processes, external factors, or their combined effects

Crowdsourced data Data collected with or without strict protocols by volunteers distributed across many locations, and placed into a repository for review and inclusion in an overall database. Advanced internet technologies are often used to harness these efforts. May also be referred to as citizen science, civic science, community science, or public monitoring data

Structured to unstructured data continuum Structured data are typically stored in tabular or relational database formats, machine readable and could be readily used in an analysis. In contrast, unstructured data are typically found in audio, image, video, or unstructured text formats, are not readily machine readable and require further specialized processing to be ready for analysis. For example, conversion and translation are needed to interpret the raw data (i.e., a target that is visible in an image or audible in a recorded sound) to an identification of the presence of an individual of a particular species. Semi-structured data fall somewhere in between, for example in xml formats where user-defined tags may be used [52]

Structured to unstructured project continuum Structured project or network designs collect data with rigorously prescribed protocols and tightly controlled measurement error, ideally with randomization to ensure representation of the overall population, and are implemented for a specific purpose or planned data analysis with clear objectives. In contrast, unstructured projects or network designs collect data by open recruitment, with few rigorous protocols, and with typically large variation in data quality and quantity within the network. Semi-structured projects fall somewhere in between, for example, by collecting information on potential covariates or biases that can be accounted for in later analysis (sensu [53, 54])

Movement Ecology Paradigm Nathan et al. [42] proposed this paradigm to organize individual movement research, based on four mechanistic components of organismal movement: (1) internal state, (2) motion, or (3) navigation capacities of an individual, and (4) external factors affecting movement 
by scale-dependent external factors, including biotic interactions, resource environments, climate change, and anthropogenic disturbance [26, 38]. In some cases, such as migration and nomadism, the different types of population-level movement roughly overlap with types that can be observed in individuals [37], whereas other types of population-level movement, such as range shifts, emerge from aggregate, long-term changes in individual behavior and demographic processes.

\section{Research themes}

We propose grouping population-level movement research into four thematic research areas: (1) Quantify population-level patterns of movement, (2) assess how species traits influence population-level movement (i.e., internal factors), (3) study how population-level movements correlate with external factors, and (4) connect movement patterns with conservation or management schema (i.e., implications and applications) (Box 2).

\section{Occurrence data}

Most sensor-network occurrence data fall somewhere along the continuum of structured to unstructured data [52], and of structured to unstructured projects (Box 1, $[53,54,58])$. From a big data perspective, occurrence data generated by human or passive automated sensors may be structured as tabular or database formats that are easily machine readable (analysis ready), unstructured as audio, image, video or text files that are not easily machine readable, or fall somewhere in between as semi-structured data [52]. From a study design perspective, projects that generate occurrence data range from structured to unstructured sensor networks. Structured projects use rigorously prescribed protocols, implement systematic or random sampling to ensure locations are representative of a larger population of interest, and are implemented for a specific purpose or planned data analysis with clear objectives $[53,54]$, for example, long-term standardized projects like the UK Butterfly Monitoring Scheme [59] or national weather surveillance radar systems [60]. In contrast, unstructured projects are collected by open recruitment, with few rigorous protocols, and typically exhibit large variation in data quality and quantity (e.g., iNaturalist, [61]). Semi-structured projects fall in between [53, 54, 58]. Data resulting from semiand unstructured projects are not typically designed to answer specific research questions [53], or to adhere to particular statistical or study design principles but are often collected with the goal of sampling a large portion of the total population within a defined area [40].

Box 2 Thematic research areas and specific research questions that are important to the emerging field of population-level movement ecology

\footnotetext{
Example research categories and question types

Research Theme 1: Quantify population-level patterns of movement

1. How does the geographic center of a population change seasonally and through time? What is the distance covered, rate of temporal change or speed, directionality, and intra- and inter-annual variation? What is the timing of migration and how does the distribution of a population change during migration?

2. How does the location of range boundaries or population clusters within a species' range, change seasonally and through time?

3. How does the population's movement compare to other populations or species?

Research Theme 2: Assess how species traits influence population-level movement

4. How is population movement constrained or facilitated by average behavioral, physiological, or morphological traits of the species?

5. For migratory species, how do migration strategies (e.g., partial, full, differential, irruptive), migration distance, morphology (e.g., body mass), or behavior (e.g., diet) impact movements?

6. To what extent are observed differences among species explained by their traits?

Research Theme 3: Study how population-level movements correlate with external factors

7. Which external factors (ecological, environmental, geographic, or anthropogenic) correlate with population-level movement? How and where do populations move in relation to these external factors?

8. What are the most relevant spatial and temporal scales for biotic or abiotic interactions to impact movement?

9. Can we develop empirical mechanistic models of population-level movement based on the observed occurrencees and external factors?

Research Theme 4: Connect movement patterns with conservation or management schema

10. How does the population's movement or the movement of it's range center or edges contribute to or change biodiversity patterns? 11. What environmental or landscape factors are needed to maintain or improve population movement efficiency or to reduce risk during movement? How are the consequences of global change (climate change, land-use change, and environmental pollution) affecting, or forecasted to affect, population-level movements?

12. Do movement trends and associations with environmental drivers suggest changes to location or range that could help guide priority concern or management strategies? Are there natural or anthropogenic barriers to movement that might be important when considering conservation under changing climate, where species may seek to move to colder areas at higher latitudes or elevations?
} 
Unstructured and semi-structured project examples often include human sensor networks such as eBird [36] and eButterfly [62], or other crowdsourced data platforms relying on professional or volunteer observers [43, 63 ] that may or may not collect effort covariates designed to account for potential sources of bias in the data [58, 64]. Structured project examples often include passive automated sensor networks that are designed by a central institution (e.g., weather surveillance radar as part of a national weather monitoring system). Structured projects may collect structured, semi-structured, or unstructured data, and the same is true for unstructured projects. The proliferation of occurrence datasets and projects that vary from structured to unstructured provides new challenges and opportunities for observing and estimating population-level movements across broad spatial scales.

Crowdsourced data can leverage the interests or expertise of many individuals to collect relatively high-density occurrence data across broad geographic areas and at high frequency through time. Many such efforts have gained traction with general community members and provide rich, and often publicly available, data sources [65]. The applications or websites on which volunteers enter occurrence information can provide some structure to the data, while allowing for flexibility in observer expertise and motivation (e.g., a casual observer who largely ignores protocols to an observer that is dedicated to following strict protocols). Many platforms for crowdsourced data provide a method for "vetting" or filtering the data to address obvious data quality issues such as misidentifications. eBird (www.ebird.org) has been a leading example, capitalizing on already dedicated birding groups and hobbyists, and developing a platform that mimics the checklist format already popular among birdwatchers [36]. New analytical methods and procedures have been developed to leverage the information provided by eBird to generate reliable estimates of species occurrence [66]. Other datasets focus on different taxonomic groups and geographic regions, but are increasingly providing the quality, density, and frequency of human-observed data necessary to assess populationlevel movements (Table 1). Appropriate use of crowdsourced data requires careful consideration of imperfect and variable detections as well as spatiotemporal variation in sampling intensity, with observations often biased towards easily accessible locations containing unique or abundant species [64, 65, 67-69].

In addition to the crowdsourced data provided by direct observations, new technologies are expanding opportunities for automated observation networks, which can be used to document occurrences and infer population-level movements. Examples of automated networks include weather surveillance radar (WSR), acoustic monitoring, and camera traps. WSR stations were developed for the purpose of monitoring precipitation, but such data streams provide new opportunities to monitor biological populations (e.g., $[79,80])$. These data may be used to monitor specific taxonomic groups (e.g., birds, bats, or

Table 1 Examples of occurrence datasets that are publicly available or can be accessed through a registered user account

\begin{tabular}{|c|c|c|c|c|}
\hline Example occurrence dataset & Sensor type & Taxa & Spatial extent & Temporal extent \\
\hline iNaturalist; https://www.inaturalist.org [61] & Crowdsourced human observers & Any & Global & 2008-present \\
\hline $\begin{array}{l}\text { Global Biodiversity Information Facility } \\
\text { (GBIF); https://www.gbif.org/ [70] }\end{array}$ & $\begin{array}{l}\text { Professional, governmental, and crowd- } \\
\text { sourced human observers }\end{array}$ & Any & Global & 2001-present \\
\hline eBird; https://ebird.org/ [36] & CROWDSOURCED human observers & Birds & Global & 1800-present \\
\hline $\begin{array}{l}\text { Herpmapper https://www.herpmapper. } \\
\text { org/ [71] }\end{array}$ & Crowdsourced human observers & Herptiles & Global & 2013-present \\
\hline eButterfly; https://www.e-butterfly.org/ [62] & Crowdsourced human observers & Lepidoptera & North America & 2011-present \\
\hline $\begin{array}{l}\text { UK Butterfly Monitoring Scheme; https:// } \\
\text { ukbms.org }[59,72,73]\end{array}$ & $\begin{array}{l}\text { Volunteer, professional, and governmental } \\
\text { human observers }\end{array}$ & Lepidoptera & United Kingdom & 1976-present \\
\hline $\begin{array}{l}\text { United States weather surveillance radar } \\
{[74,75]}\end{array}$ & Weather surveillance radar & Aerofauna & North America & 1991-present \\
\hline $\begin{array}{l}\text { European weather surveillance radar; } \\
\text { OPERA }[60,76]\end{array}$ & Weather surveillance radar & Aerofauna & Europe & 2012-present \\
\hline $\begin{array}{l}\text { North American Bat Monitoring Program; } \\
\text { [77] https://www.nabatmonitoring.org/ }\end{array}$ & $\begin{array}{l}\text { Professional and governmental acoustic } \\
\text { surveys }\end{array}$ & Bats & North America & 2009-present \\
\hline $\begin{array}{l}\text { Snapshot USA (eMammal); } \\
\text { https://emammal.si.edu/snapshot-usa [55] }\end{array}$ & Crowdsourced camera traps & Terrestrial mammals & United States & 2019-present \\
\hline $\begin{array}{l}\text { FroglD; https://www.frogid.net.au/ } \\
{[56,78]}\end{array}$ & $\begin{array}{l}\text { Crowdsourced human observers via } \\
\text { acoustic app }\end{array}$ & Frogs & Australia & 2017-present \\
\hline
\end{tabular}

The temporal extent is noted for each dataset, though it is important to recognize that most of these efforts have a significant "ramp up" period, and the frequency and quality of data from the earliest years may not be high enough to support broad-scale analyses. This list is not exhaustive and is meant to illustrate different taxonomic examples across the globe that could be used to infer population-level movement 
insects) but the data typically cannot be parsed into individual species [81-83]. Exceptions include species that occupy large roosting sites during the night, whose dawn departures can be detected by WSR [84, 85]. Significant technical knowledge is often required to screen and process WSR data [86]. After processing, WSR data may be capable of providing relative densities of biological targets as altitudinal profiles of density, speed and direction $[87,88]$. Like crowdsourced occurrence data, WSR data cannot track specific individuals, but it can provide a cost-effective density-based estimate of the distribution and movement of aerofauna across space and through time. There are several national and international WSR networks that provide data openly, or in agreement with specific research groups. WSR data is freely available in the United States (i.e., [74]) and in Europe through a multinational data exchange program (OPERA, $[60,76]$ ). Although some data and methods for parsing biological entities have also been published (e.g., MistNet, [89, 90]), acquiring and analyzing WSR data across other geographic and political regions may be more challenging due to WSR coverage gaps, limited data accessibility, and interoperability of data streams across stations [80, 91, 92].

Automated sensor networks may also include audio or visual technologies, such as acoustic monitoring and camera traps, and are sometimes deployed or maintained using crowdsourced volunteers (e.g., [46, 77, 93-95]). Camera trap and acoustic sensors may vary in their ability to isolate likely image or audio targets, may be patchily distributed across the landscape, and users may need to rely on automated software tools that are not $100 \%$ accurate or require time-consuming manual vetting processes [96]. Thus, one challenge with acoustic and camera trap data is the combination of technical software and skill needed to identify and isolate the correct sounds or images for analysis [97], but also the human time that is often needed to manually validate portions of the data for accuracy $[96,98,99]$. Sparse arrays of acoustic or camera monitors may be useful for confirming a species' occupancy, or for estimating animal activity patterns, abundance, or species diversity in an area, especially when robust methods for confirming species presence have been developed [100, 101], but much larger and denser arrays would be needed to infer population movement through or within an area [46]. Differences in camera trap survey designs, including baited versus unbaited stations, have been found to have significant consequences for occurrence frequency and detection rates [102, 103]. However, there are several examples of such arrays that have been used to infer population movement-acoustic recordings for bat occupancy trends across space and through time [104], camera traps for raptor prevalence and migration [46] and migration timing and speed of caribou and ptarmigan [105]. Although acoustic monitoring is most frequently used to observe species within a local area or during non-movement periods, it has also been used to detect bird populations during migration [106] or to "catch" the short flight calls that birds emit during migration [107]. Decreasing costs of Autonomous Recording Units (ARUs) and camera units may increase the feasibility of deploying these sensor technologies to detect and infer population movement in future studies $[100,108]$.

Due to the partially unstructured nature of data, common for all of these networks, these datasets require a significant time investment to process and validate data, and careful consideration of possible sampling biases and variability in an observer's skill before they can be used for analysis. A benefit for population-level movement research is that much of these data are publicly available (Table 1) or are available upon request, in contrast with individually tracked data, which may be more likely to be protected and only accessible within specific labs or institutions (but see Movebank's data repository for a library of openly published individual track data; https://www. datarepository.movebank.org/). Data from crowdsourced human and sensor networks are most readily available at appropriate densities from the northwestern hemisphere, specifically from North America and Europe, and have a strong bias towards aerofauna, including birds, flying insects, and bats.

\section{Analytical approaches to estimate population-level movement \\ Data processing}

Prior to statistical analysis, occurrence data frequently require cleaning and processing. Data processing methods may differ among datasets and for distinct research questions, but general challenges include estimating occupancy from presence-only data [109], standardizing sampling intensity by subsampling observations (e.g., [67, $110]$ ), accounting for low detection probability of certain species or in certain time periods or habitats [102], the potential for false positive occurrences [96], and accounting for detection or sampling biases related to human behavior [66]. Clear guidelines or code to aid in processing the data may be available for some datasets, or may require more technical knowledge to navigate, especially for WSR, acoustic, or image data [89]. In other cases, common clear methods may not exist, and a researcher may need to develop their own data processing workflow using appropriate analysis techniques that account for imperfect detection or variable sampling effort (Fig. 3). 


\section{DATA SELECTION}

\section{Unstructured}

\section{Structured}

Most occurrence data begin as unstructured or semi-structured, with study designs and protocols that range from loosely to highly structured. Data processing helps add structure by controlling for quality and adjusting for variable and biased sampling effort.

\section{DATA PROCESSING}

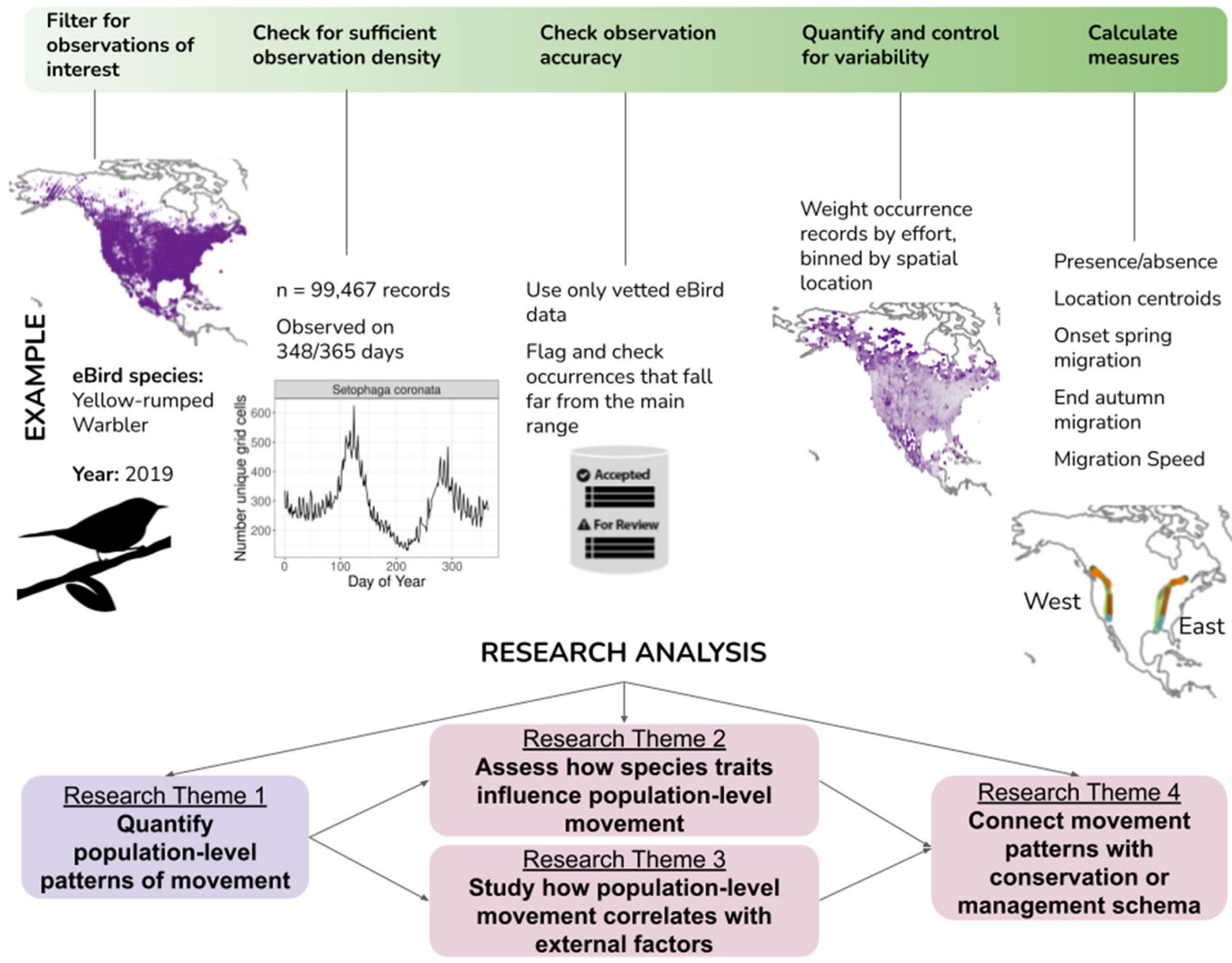

Fig. 3 Schematic of the steps from data selection to data processing and analysis that could be used to evaluate population-level movement from occurrence data. An example is shown using eBird occurrence data from the western and eastern flyways of the Yellow-rumped Warbler (Setophaga coronata) in 2019 [36], but the same general workflow could be applied to other occurrence datasets. Yellow-rumped Warbler silhouette was created by Cornell Lab of Ornithology and is used with permission

\section{Data vetting and cleaning}

Technical and logistical challenges exist for researchers using semi-structured or unstructured data, as data are often not ready "out of the box" for analysis, and because different data and project structures require unique statistical approaches to minimize bias [53, 111, 112]. Each occurrence dataset will contain different fields, constraints on data collection, variability among sensors or observers, and the oversight or protocols behind a given project. These factors need to be accounted for to reduce bias and avoid flawed conclusions (Fig. 3) [58, 65]. Variability in data collection and data quality can often be dealt with in the data processing stages but may also need to occur within the data analysis stage. When preparing occurrence datasets for movement analysis it should be useful to follow some 
or all of the checks presented below for filtering data, checking for coverage, accuracy, and accounting for variability.

\section{Filter for observations of interest}

For any occurrence dataset, an initial first step is often to parse out observations that represent biological entities, the target specie(s) and/or locations of interest, and observations that have a high likelihood of being accurate (e.g., not a misidentification). For WSR and other automated sensor network data such as acoustic or camera arrays, simply identifying records that represent biological entities, and then filtering to the target species or taxa is itself a non-trivial process (e.g., [113, 114]). Many tools have been developed to screen and process WSR data for biological information including the Bias Improvement of Radar Data System (BIRDS; methods described in [115]), and computational packages such as w2birddensity (part of WDSS-II [116]), vol2bird [87], R package bioRad, [117], and MISTNET [89]. Camera trap and acoustic data research also increasingly rely upon artificial intelligence systems to automate and speed the process of filtering for target biological entities [97, 118-122], possibly using image or acoustic libraries (e.g., [123]). But such data still often require a significant investment in manual humanverification (e.g., [98]) before the data can be used for analysis.

\section{Check for sufficient observation density and trends over time}

To infer movement, the data need to have adequate coverage across space and time. Determining if there is sufficient data can be done in multiple ways, but often requires simply exploring patterns or structure in the data across time and space (e.g., using binned data). Simple visualizations or descriptive summary statistics may help the researcher determine if patterns occur due to imbalances in sampling, survey effort, or data collection methods, or represent valid ecological patterns. Strong increasing trends in total occurrences, the number of spatial locations observed, or the number of time frames observed may indicate that a data set is undergoing strong growth and should be used to infer movement with caution, or that it requires the application of a subsampling or weighting method as a first data processing step (e.g., [124]) (Fig. 4). Less pronounced increases or plateaus in the occurrence trends may indicate more stable data collection across space and time (Fig. 4). Controlling for overall sampling effort, spatially or temporally binning occurrences, and resampling or subsampling methods may all be useful ways to control for variability in sampling effort when analyzing occurrence data. In some cases, the researcher may decide to drop certain time frames or spatial areas from analysis if they do not meet a minimum threshold of occurrences for analysis (inclusion criteria; e.g. see methods from [124]).

\section{Check observation accuracy}

Data veracity is a challenge for many occurrence datasets, and substantial variability in data quality may exist, including the existence of false positive or false negative records. Such inaccuracies, misidentifications, or imperfect detections are mainly a problem if they change significantly over space or through time. Fortunately, many occurrence datasets have protocols in place for human experts to validate questionable observations. For example, programs like eBird, Project FeederWatch, and HerpMapper use automated processes [126] and expert review $[36,71]$ to validate the data. Expert review may also be used to validate signals resulting from automated classification processing of acoustic or camera sensors [98, 99]. When using occurrence data, a researcher should first review the guidelines for individual datasets (e.g., [66, 127]) or view protocols or methods from recent publications that address potential sources of inaccuracy in the data (e.g., false-positive occupancy models [96]). When guidelines do not yet exist, researchers may need to rely on expert knowledge to look for and filter outliers or suspect records on their own. A researcher may want to consider the species- or habitat-specific context for their research subject and use their own protocols to filter occurrence records outside the known species range or cases where the species is known to have low detection probability or high misidentification rates (e.g., in the case where two similar species co-occur). Often, occurrence data are used to quantify occupancy (presence within a spatial area or grid cell) or relative abundance. Although abundance estimators are sometimes used with occurrence data, these methods often require strong assumptions (e.g. population closure, no double counting, and constant detection probability) that are difficult to verify and can significantly impact estimator accuracy [128-130].

\section{Quantify and control for variability}

Perhaps the largest challenge is to acknowledge and account for sources of variability that originate from the observation process (e.g., variability in sampling effort or observer skill level) within occurrence datasets and control for it where possible. Substantial variability in data quality, data volume, and overall sampling effort across time and space are characteristics of "big data" [52, 65] that are also common to occurrence data. Data fields that record sampling effort, sensor placement, factors related to detection probability, and spatial and temporal 

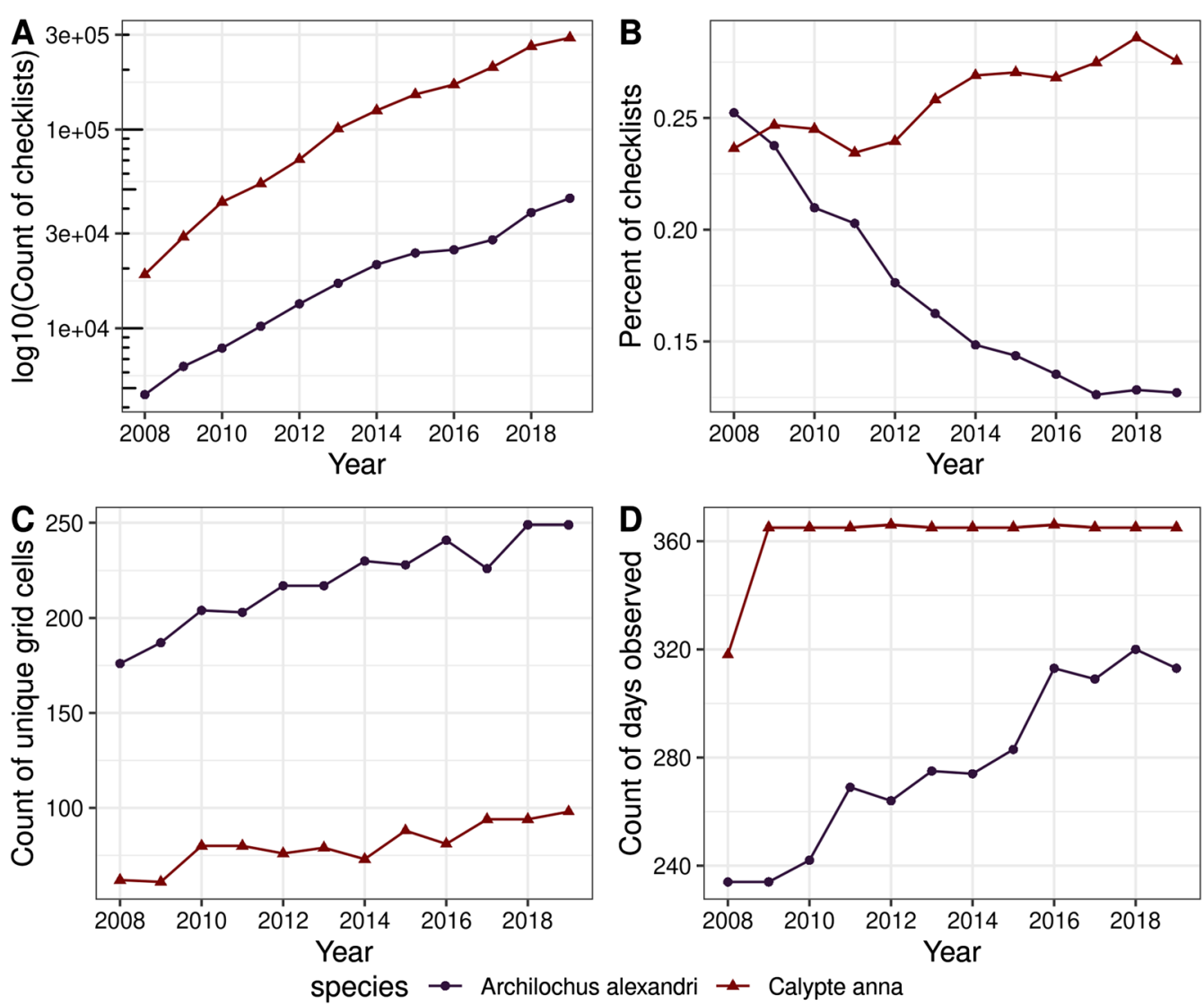

Fig. 4 A worked example exploring observation trends in eBird occurrence data from 2008 to 2019 for two closely related species: migratory Black-chinned Hummingbird (Archilochus alexandri) and range-expanding Anna's Hummingbird (Calypte anna) [125] from the western flyway of North America [36]. Even closely related species can display different dynamics, which can dramatically affect how the data is structured across space and time. (A) The total number of checklists ( $\log _{10}$ transformed) containing each species increases through time, which is expected as the crowdsourced platform gains new observers. It does not represent an increase in the total number of hummingbirds. (B) In contrast, the percent of all checklists containing each species within regions where each occurs is declining for Black-chinned Hummingbirds, and increasing for Anna's Hummingbirds, which may reflect changes in observer behavior, expertise, or geographic coverage through time. (C) After spatially binning the data, the number of unique grid cells in which each species is observed increases slightly through time, but is relatively flat in recent years, giving some confidence that the species' locations have been adequately covered through the time frame and within the spatial area. (D) The number of days that the species was observed in each year is flat for Anna's Hummingbird after 2008, indicating that they were observed every day in each subsequent year. In contrast, Black-chinned Hummingbirds show a strong increasing trend, which suggests a need to further explore the data to see whether it indicates increased observer effort in general, or at particular locations or times of the year, or if it represents a meaningful ecological trend in the occurrence phenology of the species

resolution of the data, should be collected and included in models to help control for variation when possible. Other effort-based measures may be used, such as time spent searching, distance traveled while searching, number of observers in the search party, or time of day (e.g., eBird [36]). For camera traps or acoustic sensors, effortbased measures could include fields for the number of days a camera/acoustic sensor was active, whether it was baited or not, or if sensors were placed randomly or chosen opportunistically to increase detection, for example, by focusing on known travel routes or previous occurrence locations [103]. Such measures can help to control for crowdsourced effort differences across time and space, rather than using the raw occurrence data.

Recent work has focused on developing methods and approaches that account for variable and spatially biased sampling effort, sometimes by integrating data from multiple structured and/or unstructured projects [131-133], binning the data spatially and/or temporally ([28], e.g., [134]), or weighting records [135]. For example, data can be standardized within a spatial bin by accounting for overall sampling effort (e.g. the total number of observers). Effort measures can be used to weight records, as predictors in an analysis, or in some cases, modeled using 
an offset (a predictor with a regression coefficient $=1$, so that a count-based model effectively models density or encounter rate, [136]). In some cases, rarefaction or resampling methods (i.e., oversampling or undersampling to a median value [124]) may be used to represent a similar sampling effort across space or through time. Variable sampling effort over space is often accounted for using covariates that are suspected to correlate with sampling effort, such as distance to the nearest urban center (e.g., [137]) or distance to road [68]. In some cases effort covariates do not exist (e.g., iNaturalist [101]) and researchers must instead control for variable sampling effort with other methods, such as using the number of non-target species detections as a way to estimate change in effort across space and time [138]. Another common approach used when fitting species distribution models is to sample background locations in a way that attempts to mimic sampling biases in the occurrence data $[139,140]$. Alternatively, setting strict inclusion criteria may help to deal with variability by omitting extreme or outlier observations or spatial or temporal bins that do not meet a predetermined threshold for total number of occurrences.

Ultimately, variation in data and project structures makes interoperability across datasets challenging. A major goal for researchers should be to identify better ways to coordinate data collection efforts, to link data observations across different collection sites and platforms, and to develop automated tools to make this process accessible to researchers at different computational skill levels and from different subfields. Species distribution modeling represents one area of research that has developed methods to integrate different types of occurrence data (e.g. presence-only, presence/absence, and count), that might prove useful for aggregating occurrence data to infer population-level movements or the causes of those observed movements [131-133, 141, 142]. Improved standards for data collection and project structure, and adherence to data sharing policies across institutional, national, and international boundaries would benefit the utility of occurrence data for movement ecology [80], considering that animal movement research often requires monitoring at regional, continental, or global spatial scales.

\section{Methods and models}

\section{Statistical methods}

Population-level movement can be inferred from occurrence data in multiple ways: (1) A summary of predicted occurrence distributions at multiple points in time can be modeled to evaluate change (e.g., in the center or boundaries) over time, and (2) changes in the occurrence distribution can be directly modeled as a function of temporally changing explanatory variables. Statistical processes for inferring population-level movement from semi- and unstructured occurrence datasets and projects must be able to account for a high volume of data that contain variable quality, noise, recording anomalies, spurious correlations and incidental endogeneity throughout [52, 143]. Incidental endogeneity is a genuine relationship between predictor variables and the error term in a regression analysis (i.e., residual term is dependent on some of the predictors), and is common in observational data and in highly dimensional data that comes from multiple different sources, such as crowdsourced data $[52,112,143]$. Occurrence data also frequently violate common statistical assumptions of independence, stationarity, and Normality [40]. Spatial [144-146] and temporal $[147,148]$ autocorrelation are common concerns. Additional challenges include finding ways to deal with multicollinearity (i.e., when an explanatory variable can be predicted by linear combinations of other explanatory variables $[149,150])$ or overfitting due to excessive model flexibility (e.g., too many predictors or too flexible a relationship between predictor and response varaible) and accidentally masking the true effects. Generalized additive models (GAMs), and tree-based and other machinelearning methods use cross-validation or penalization in an attempt to avoid overfitting. Even in the absence of multicollinearity and overfitting, models, including advanced tree-based or machine-learning methods, may still have low predictive ability when transferred to novel locations or scenarios [151]. Spurious correlations can occur, particularly when attempting to identify important species traits and environmental drivers of populationlevel movements with large datasets [143]. Measurement errors can compound, particularly when predictor variables are remotely sensed and are thus only available on coarse grids or where they are spatially or temporally mis-aligned with occurrence records. Based on the above challenges and assumptions, there is a strong need to present levels of uncertainty associated with movement models based on occurrence data.

Modeling approaches should be tailored to the data and the specific research theme and question of a given project. Some modeling approaches use only occurrence records to describe movement paths or trajectories after vetting and controlling for bias in the occurrence data itself (Research Theme 1). Others rely on additional information such as species traits, behavioral models, environmental covariates, or anthropogenic factors (Research Themes 2-4). Below, we summarize recent analytical methods and approaches for describing or quantifying population-level movement across the four research themes, with a focus on methods for Research Theme 1, describing population-level movement. 


\section{Research theme 1: quantify population-level patterns of movement}

Quantifying population-level movement is an important step to integrating population-level and landscape-scale perspectives into movement ecology, which to date has largely focused on the movements of individuals. Crossing or combining organizational scales to understand the changes to population-level measures of distribution that emerge from individual processes is a growing area of study that can provide new insights. Due to the nature of occurrence data, population-level movement is usually characterized using quantitative measures of the population's range area, range edges, or range center, and changes in these characteristics through time. For populations undertaking directional movement through migration, or undergoing range expansion or range shifts, measures may describe location, direction, density of moving individuals (migration traffic rate, [152]), or speed (e.g., $[28,105])$ over a time period, or compare differences in location, density, or velocity across multiple time periods and across space (e.g., [153, 154]). Estimates of the timing of events, such as date of first arrival, crossing a latitudinal or longitudinal demarcation, or reaching a predetermined number or density of observations (e.g., date when half the population has passed a sample point $[105,155])$, may also be used to infer or evaluate population-level movement.

One may characterize the central location and movement of a population using coarse summaries of the occurrence data, such as the population's centroid in latitude and longitude for a given time period (e.g. daily or weekly). The population centroid can then be compared across time or thresholds that indicate movement $[28,105]$ or arrival at a predetermined area (e.g., [155]). Changes in centroids can be modeled using generalized additive mixed models (GAMM, [156]) or any regression-based approach, allowing users to estimate when migratory species begin or end directional movement (e.g., the onset of spring migration or the end of autumn migration). Further, measures of distance, speed, and direction of movement across seasons or years can be calculated from measures of population centrality. For example, population migration speed may be calculated by measuring the distance between daily centroids as $\mathrm{km} /$ day (birds 28) or km/hr (caribou 99) traveled. For longerterm occurrence datasets, these approaches are able to provide a description of population movement through annual life cycles, while accounting for variability among years using random effects (e.g., [134]).

Robust adaptive spatio-temporal models (AdaSTEM), a form of ensemble species distribution models, have been developed for use with eBird data. These models automatically select the appropriate sized stixel (spatio-temporal block of data) for inferring occurrence and abundance across a region based on the quantity of observations $[124,157]$. These models do not measure movement, per se, but their estimates of occurrence, abundance, and range can be compared to evaluate how populations move based on shifting species distribution ranges and centers over the target time period. AdaSTEM models are useful because they are semi-parametric and can be used to generate hypotheses for migration dynamics and range expansion, or dispersal across dynamic species ranges, as is necessary especially for migratory species [67]. Estimates of ranges can be compared across different times of the year, or across years, to infer population movement [124]. Scientists working with eBird data have led the development of many new statistical methods and tools for conducting movement research using occurrence data. These new approaches have been successfully applied to eBird data, which contain a large amount of high quality, vetted occurrence records for birds, across broad geographic areas for more than 10 years $[36,66]$. Nonetheless, some aspects of these eBird-inspired workflows and statistical methods could be reasonably adapted to other crowdsourced or occurrence datasets, different taxonomic groups, and other types of population movement research questions.

Rather than use a two-step approach (estimating species distributions at different time points and then modeling summary measures of these distributions, e.g. their centrality), it is appealing to consider more mechanistic approaches that model movement in terms of an advection-diffusion process [158]. This approach has been successfully applied to model the spread of Eurasian Collared-Dove (Streptopelia decaocto) using structured breeding bird survey data [55], but we are unaware of any successful applications of diffusion models to unstructured project survey data. The modeling approach of Wikle [158] is an example of a state-space model, with separate models for the underlying biological movement and observation processes. Alternative formulations of state-space models could be considered for quantifying population-level movements, but these methods require a significant understanding of advanced mathematics and access to high-level computing tools or high-performance computing systems to fit these models to large datasets [159].

\section{Research themes 2 and 3: evaluating the effects of species traits or external factors on movement}

Once population movement has been characterized, a logical next step is to assess possible traits or external factors driving movement Outputs from the previous step that describe population-level movement can be used to examine predictors or correlates of 
movement and to test relevant questions or hypotheses. Since population-level movements are often summarized using an aggregate measure of the population (e.g., its center or range boundary), predictors should be expected to influence a significant number of individuals across a large spatial or temporal scale. Predictor variables frequently come from other datasets, for example, remote sensing products (e.g., NASA MODIS, LANDSAT, landcover or anthropogenic databases, other species occurrence records) or climate reanalyses (e.g., Daymet, NCEP, ECMWF, ERA5), or trait databases [160-162]. These predictors can be analyzed with population-level movement metrics using generalized linear mixed models that account for different sources of variation in the data using combinations of random and fixed effects [163, 164]. In some

Box 3 Examples of new ecological insights that have been gained in each thematic area with citations

\section{Ecological insights}

Research Theme 1-Quantify population-level patterns of movement

- Broad scale migration patterns, including looped migration, and directionality $[28,88,134,152,165,166]$

- Migration timing, including when animals migrate and how quickly they migrate $[47,92,105,155,167,168]$

- Estimates of range expansion or contraction, overall shift in center, area, or edges of range $[56,169]$

Research Theme 2-Assess how species traits influence population-level movement

- Species traits impact range expansion [32] and range shifts during periods of rapid climate change

[170]

- Species traits (body mass, total migration distance) impact avian migration patterns [28]

- Species migratory traits affect sensitivity to migration phenology [171]

Research Theme 3-Study how population-level movement correlates with external factors

- Distance in range shift relative to temperature change, climatic debt [33], and climate velocity during periods of long-term climate transition [170]

- Importance of topography and tailwind for migration [87, 152]

- Environmental correlates of migration including atmospheric conditions [172], temperature $[62,154]$, and ecological productivity [173,

174]

- Assess whether species presence or absence across sites is affected by other species presence relative to timing of migration [46]

Research Theme 4-Connect movement patterns with conservation or management schema

- Association of migratory birds with protected areas and land-cover categories across the annual cycle $[175,176]$

- Impacts to moving species from projected changes in climate and land use [177]

- Impacts to society from range movement or redistribution due to climate change [178]

- Potential environmental barriers to migration [153]

- Urban effects on occurrence of birds and mammals [179-181]

- Conservation planning based on movement and abundance across species' annual cycles [182] cases, machine learning based models (i.e., random forests) may be able to identify the relative importance of different predictors for movement and/or changes in occurrence (e.g., [154]).

\section{Research theme 4: connect movement patterns with conservation or management schema}

Most conservation applications will apply an understanding of how distributions and movement are influenced by species-level or environmental covariates to a particular location or set of locations. In other words, Research Theme 4 requires the methods from Themes $1-3$, but with a further step to translate the science to decision-making, and to influence on-the-ground management. From a robust description of population movement, ideally including some analysis of species traits or external factors, it becomes possible to forecast the effects of anthropogenic and environmental changes on future movement corridors or population distributions and to develop conservation and management strategies.

\section{New ecological insights}

Widespread occurrence data and new analytical approaches have allowed scientists to describe large-scale population movement, compare patterns among species or regions, and uncover potentially useful new strategies for conservation in ways that have not always been possible, or may have been very constrained, using only tracking data from focal individuals. Using measures of population-level movement and its correlates, researchers have uncovered broad-scale patterns across species' annual life cycles, and quantified general relationships between population movement, expansion, or contraction and species traits and environmental associations (Box 3).

\section{Conclusions and next steps}

The rapid growth in occurrence data as well as new computational tools and statistical methods have opened the door to new possibilities for inferring population-level movement across broad spatial scales. While scientists at eBird and those specializing in analysis of WSR data have thus far led the development of datasets and new statistical methods and tools for analyzing movement from occurrence records, many of these approaches could be reasonably adapted to other crowdsourced or occurrence datasets, different taxonomic groups, and other types of research questions addressing population-level movement. Population-level movement studies have previously focused on data from individual studies that represent a relatively small sample of larger populations, but 
growing networks and increasingly available and interoperable data make larger collaborations and advances possible. Work that integrates different data sources and includes both individual- and population-level movement metrics (e.g., [49, 183-185]) have the potential to create models that share parameters across data, locations, and spatial or temporal resolutions to provide a more comprehensive summary of animal movement. Further, process-based modelling using individual tracking datasets could prove useful for testing hypotheses derived from occurrence data and for developing insights into alternate mechanisms driving observed populationlevel movement patterns.

As sensor networks for occurrence data mature, current limitations will hopefully be addressed through increased data quantity, improved methods for estimating accuracy and bias, and enhanced metadata standards. Population-level movement studies are "data hungry"sufficient thresholds of the number of occurrences across space and time are needed to conduct a meaningful analysis - and not all datasets have the appropriate spatiotemporal coverage or volume of records required to make reliable estimates of population movement. Finally, the development of common standards for occurrence datasets could aid in increasing data availability, accessibility, and interoperability, as well as facilitating more robust adjustments for sampling effort and bias. For example, not all crowdsourced data repositories collect the necessary information to account for variation in observer skill or sampling effort, but an endeavor to do so would improve the utility of these data for movement ecology, and other scientific research. In some cases, related parts of sensor networks differ in their data accessibility or the resolution at which key geographic, temporal, or taxonomic variables are recorded, which can make it challenging for researchers to use data across broader spatial scales.

Currently, population movement studies are strongly biased towards birds, followed by other types of aerofauna such as bats and insects, and are geographically biased to North America and Europe. These biases make clear the exciting opportunities for new research in this area. There is high potential for new ecological insights to be gained as data are collected across other parts of the planet and for other taxonomic groups. This review only examined research related to terrestrial animal movement, but many of the same concepts and approaches can be applied to populations of aquatic and marine (e.g., [186, 187]), microbial [29], and plant taxa, which move over generations by natural or human-assisted dispersal (e.g., [188-190]. Using these data and new statistical methods to assess population-level movement can help support current work being carried out with individual tracking to determine how individual movement observations fit within the whole of a population or species' trajectory and average patterns of movement. In addition, a population-level perspective can help shed new light on large-scale macro-movement patterns and associations with biological and environmental correlates of movement that might not be as obvious when considering the variation between specific individuals that were sampled (Fig. 1). Finally, documenting population movement may help scientists gain a clearer macroecological understanding of species occurrence, range expansion or contraction, migration, and needs for conservation and management in a changing world.

\section{Acknowledgements \\ SRS and GB were supported by the National Science Foundation awards 1915909 and 1915913. GB and JF were supported by NASA award 80NSSC21K1182. JF received partial salary support from the Minnesota Agri- cultural Experimental Station. The authors wish to acknowledge the valuable efforts of the many scientists and volunteers who have collected and continue to collect broad scale occurrence data. We also wish to acknowledge the contributions of the two anonymous reviewers and the associate editor who provided valuable feedback to improve the manuscript.}

\section{Authors' contributions}

SRS and GB conceived the review. SRS drafted the manuscript. SRS and FAL created figures for the manuscript. All authors discussed, edited, read, and approved the final manuscript.

\section{Authors' information}

SRS, is an Assistant Professor of Data Analytics at Denison University, where she teaches interdisciplinary data science skills and conducts research in macroecology, biodiversity change, and animal movement.

$\mathrm{GB}$, is an environmental engineer and models the effects of environmental conditions on atmosphere-biosphere exchange, and animal movement. He is one of the developers of the Env-DATA toolpack.

JF, is an Associate Professor of Quantitative Ecology at the University of Minnesota where he teaches courses in statistics. His research interests focus on the development and application of robust statistical methods, with an emphasis on quantifying species-habitat associations.

$F A L$, is a research ecologist at the Cornell Lab of Ornithology where he studies questions related to the macroecology, biogeography, and conservation of birds and plants within the context of global environmental change.

\section{Funding}

SRS and GB were supported by the National Science Foundation awards 1915909 and 1915913. GB and JF were supported by NASA award 80NSSC21K1 182. JF received partial salary support from the Minnesota Agricultural Experimental Station.

\section{Availability of data and materials}

The datasets used to create figures or examples in the current review are cited within the text and the references and are freely available for download or from the data collector upon reasonable request.

\section{Declarations}

Ethics approval and consent to participate Not applicable.

Consent for publication

Not applicable.

Competing interests

The authors declare that they have no competing interests. 


\section{Author details}

1 Data Analytics Program, Denison University, Granville, OH 43023, USA ${ }^{2}$ Department of Civil, Environmental and Geodetic Engineering, The Ohio State University, Columbus, OH 43210, USA. ${ }^{3}$ Department of Fisheries, Wildlife, and Conservation Biology, University of Minnesota, Minneapolis, MN 55455 , USA. ${ }^{4}$ Cornell Lab of Ornithology, Cornell University, Ithaca, NY 14850, USA.

Received: 10 June 2021 Accepted: 11 November 2021

Published online: 11 December 2021

\section{References}

1. Soulsbury CD, lossa G, Baker PJ, White PCL, Harris S. Behavioral and spatial analysis of extraterritorial movements in red foxes (Vulpes vulpes). J Mammal. 2011:92(1):190-9.

2. Supp SR, Koons DN, Ernest SKM. Using life history trade-offs to understand core-transient structuring of a small mammal community. Ecosphere. 2015;6(10):art187.

3. Van Moorter B, Rolandsen CM, Basille M, Gaillard J-M. Movement is the glue connecting home ranges and habitat selection. J Anim Ecol. 2016;85(1):21-31.

4. Friedemann G, Leshem Y, Kerem L, Shacham B, Bar-Massada A, McClain $\mathrm{KM}$, et al. Multidimensional differentiation in foraging resource use during breeding of two sympatric top predators. Sci Rep. 2016;6(1):35031.

5. Ahearn SC, Dodge S, Simcharoen A, Xavier G, Smith JLD. A context-sensitive correlated random walk: a new simulation model for movement. Int J Geogr Inf Sci. 2017;31(5):867-83.

6. Bischof R, Loe LE, Meisingset EL, Zimmermann B, Van Moorter B, Mysterud A. A migratory northern ungulate in the pursuit of spring: jumping or surfing the green wave? Am Nat. 2012;180(4):407-24.

7. Bartlam-Brooks HLA, Beck PSA, Bohrer G, Harris S. In search of greener pastures: using satellite images to predict the effects of environmental change on zebra migration. J Geophys Res Biogeosci. 2013:118(4):1427-37.

8. Bohrer G, Beck PS, Ngene SM, Skidmore AK, Douglas-Hamilton I. Elephant movement closely tracks precipitation-driven vegetation dynamics in a Kenyan forest-savanna landscape. Mov Ecol. 2014;2(1):2

9. Rickbeil GJM, Hermosilla T, Coops NC, White JC, Wulder MA. Barrenground caribou (Rangifer tarandus groenlandicus) behaviour after recent fire events; integrating caribou telemetry data with Landsat fire detection techniques. Glob Change Biol. 2017;23(3):1036-47.

10. Gurarie E, Hebblewhite M, Joly K, Kelly AP, Adamczewski J, Davidson SC, et al. Tactical departures and strategic arrivals: Divergent effects of climate and weather on caribou spring migrations. Ecosphere. 2019;10(12):e02971.

11. Kays R, Crofoot MC, Jetz W, Wikelski M. Terrestrial animal tracking as an eye on life and planet. Science. 2015;348(6240). https://science.scien cemag.org/content/348/6240/aaa2478.

12. Kays R, McShea WJ, Wikelski M. Born-digital biodiversity data: millions and billions. Divers Distrib. 2020;26(5):644-8.

13. Mandel JT, Bohrer G, Winkler DW, Barber DR, Houston CS, Bildstein KL. Migration path annotation: cross-continental study of migration-flight response to environmental conditions. Ecol Appl. 2011;21(6):2258-68.

14. Davidson SC, Bohrer G, Gurarie E, LaPoint S, Mahoney PJ, Boelman NT, et al. Ecological insights from three decades of animal movement tracking across a changing Arctic. Science. 2020;6:712-5.

15. Patterson TA, Hartmann K. Designing satellite tagging studies: estimating and optimizing data recovery. Fish Oceanogr. 2011;20(6):449-61.

16. McGowan J, Beger M, Lewison RL, Harcourt R, Campbell H, Priest M, et al. Integrating research using animal-borne telemetry with the needs of conservation management. J Appl Ecol. 2017:54(2):423-9.

17. Patterson TA, Parton A, Langrock R, Blackwell PG, Thomas L, King R. Statistical modelling of individual animal movement: an overview of key methods and a discussion of practical challenges. AStA Adv Stat Anal. 2017;101(4):399-438.

18. Wikelski M, Kays RW, Kasdin NJ, Thorup K, Smith JA, Swenson GW Jr. Going wild: what a global small-animal tracking system could do for experimental biologists. J Exp Biol. 2007;210(2):181-6.
19. Gottwald J, Zeidler R, Friess N, Ludwig M, Reudenbach C, Nauss T. Introduction of an automatic and open-source radio-tracking system for small animals. Methods Ecol Evol. 2019;10(12):2163-72.

20. Hebblewhite $M$, Haydon DT. Distinguishing technology from biology: a critical review of the use of GPS telemetry data in ecology. Philos Trans R Soc B Biol Sci. 2010;365(1550):2303-12.

21. Navarro A, Oliva V, Zamorano MJ, Ginés R, Izquierdo MS, Astorga $\mathrm{N}$, et al. Evaluation of PIT system as a method to tag fingerlings of gilthead seabream (Sparus auratus L.): effects on growth, mortality and tag loss. Aquaculture. 2006;257(1):309-15.

22. Jepsen N, Thorstad EB, Havn T, Lucas MC. The use of external electronic tags on fish: an evaluation of tag retention and tagging effects. Anim Biotelemetry. 2015:3(1):49.

23. Bainbridge L, Stockwell M, Valdez J, Klop-Toker K, Clulow S, Clulow J, et al. Tagging tadpoles: retention rates and impacts of visible implant elastomer (VIE) tags from the larval to adult amphibian stages. Herpetol J. 2015;8:133-40.

24. Fieberg J, Kuehn DW, DelGiudice GD. Understanding variation in autumn migration of northern white-tailed deer by long-term study. J Mammal. 2008:89(6):1529-39.

25. Fieberg JR, Conn PB. A hidden Markov model to identify and adjust for selection bias: an example involving mixed migration strategies. Ecol Evol. 2014;4(10):1903-12.

26. Mueller T, Fagan WF. Search and navigation in dynamic environments - from individual behaviors to population distributions. Oikos. 2008;117(5):654-64.

27. Mueller T, Olson KA, Dressler G, Leimgruber P, Fuller TK, Nicolson C, et al. How landscape dynamics link individual- to population-level movement patterns: a multispecies comparison of ungulate relocation data. Glob Ecol Biogeogr. 2011;20(5):683-94.

28. La Sorte FA, Fink D, Hochachka WM, DeLong JP, Kelling S. Populationlevel scaling of avian migration speed with body size and migration distance for powered fliers. Ecology. 2013;94(8):1839-47.

29. Kottelenberg D, Hemerik L, Saponari M, van der Werf W. Shape and rate of movement of the invasion front of Xylella fastidiosa spp. pauca in Puglia. Sci Rep. 2021;11(1):1061.

30. Gallo T, Waitt D. Creating a successful citizen science model to detect and report invasive species. Bioscience. 2011;61(6):459-65.

31. Kamenova S, Bartley T, Bohan D, Boutain J, Colautti R, Domaizon I, et al. Invasions toolkit: current methods for tracking the spread and impact of invasive species. Adv Ecol Res. 2017;1:56.

32. Angert AL, Crozier LG, Rissler LJ, Gilman SE, Tewksbury JJ, Chunco AJ. Do species' traits predict recent shifts at expanding range edges? Ecol Lett. 2011;14(7):677-89.

33. Devictor V, van Swaay C, Brereton T, Brotons L, Chamberlain D, Heliölä $J$, et al. Differences in the climatic debts of birds and butterflies at a continental scale. Nat Clim Change. 2012;2(2):121-4.

34. Sunday JM, Pecl GT, Frusher S, Hobday AJ, Hill N, Holbrook NJ, et al. Species traits and climate velocity explain geographic range shifts in an ocean-warming hotspot. Ecol Lett. 2015;18(9):944-53.

35. Michel NL, Saunders SP, Meehan TD, Wilsey CB. Effects of stewardship on protected area effectiveness for coastal birds. Conserv Biol. 2021. https://doi.org/10.1111/cobi.13698.

36. Sullivan BL, Aycrigg JL, Barry JH, Bonney RE, Bruns N, Cooper CB, et al. The eBird enterprise: an integrated approach to development and application of citizen science. Biol Conserv. 2014;1 (169):31-40.

37. Teitelbaum CS, Mueller T. Beyond migration: causes and consequences of nomadic animal movements. Trends Ecol Evol. 2019;34(6):569-81.

38. Schlägel UE, Grimm V, Blaum N, Colangeli P, Dammhahn M, Eccard JA, et al. Movement-mediated community assembly and coexistence. Biol Rev. 2020;95(4):1073-96.

39. Shamoun-Baranes J, Alves JA, Bauer S, Dokter AM, Hüppop O, Koistinen J, et al. Continental-scale radar monitoring of the aerial movements of animals. Mov Ecol. 2014;2(1):9.

40. La Sorte FA, Lepczyk CA, Burnett JL, Hurlbert AH, Tingley MW, Zuckerberg B. Opportunities and challenges for big data ornithology. The Condor. 2018;120(2):414-26.

41. Estrada A, Morales-Castilla I, Caplat P, Early R. Usefulness of species traits in predicting range shifts. Trends Ecol Evol. 2016;31(3):190-203. 
42. Nathan R, Getz WM, Revilla E, Holyoak M, Kadmon R, Saltz D, et al. A movement ecology paradigm for unifying organismal movement research. Proc Natl Acad Sci. 2008;105(49):19052-9.

43. Dickinson JL, Zuckerberg B, Bonter DN. Citizen science as an ecological research tool: challenges and benefits. Annu Rev Ecol Evol Syst. 2010;41(1):149-72.

44. Blumstein DT, Mennill DJ, Clemins P, Girod L, Yao K, Patricelli G, et al. Acoustic monitoring in terrestrial environments using microphone arrays: applications, technological considerations and prospectus. J Appl Ecol. 2011;48(3):758-67.

45. Kays R, Kranstauber B, Jansen P, Carbone C, Rowcliffe M, Fountain T, et al. Camera traps as sensor networks for monitoring animal communities. In: 2009 IEEE 34th conference on local computer networks. Zurich, Switzerland: IEEE; 2009 [cited 2021 May 25]. p. 811-8. http:// ieeexplore.ieee.org/document/5355046/.

46. Jachowski DS, Katzner T, Rodrigue JL, Ford WM. Monitoring landscape-level distribution and migration Phenology of Raptors using a volunteer camera-trap network. Wildl Soc Bull. 2015;39(3):553-63.

47. Newson SE, Evans HE, Gillings S. A novel citizen science approach for large-scale standardised monitoring of bat activity and distribution, evaluated in eastern England. Biol Conserv. 2015;1(191):38-49.

48. Campos-Cerqueira M, Aide TM. Improving distribution data of threatened species by combining acoustic monitoring and occupancy modelling. Methods Ecol Evol. 2016;7(11):1340-8.

49. Gilbert NA, Pease BS, Anhalt-Depies CM, Clare JDJ, Stenglein JL, Townsend PA, et al. Integrating harvest and camera trap data in species distribution models. Biol Conserv. 2021;258:109147.

50. Mair L, Harrison PJ, Jönsson M, Löbel S, Nordén J, Siitonen J, et al. Evaluating citizen science data for forecasting species responses to national forest management. Ecol Evol. 2017;7(1):368-78.

51. Coxen CL, Frey JK, Carleton SA, Collins DP. Species distribution models for a migratory bird based on citizen science and satellite tracking data. Glob Ecol Conserv. 2017;1(11):298-311.

52. Gandomi A, Haider M. Beyond the hype: big data concepts, methods, and analytics. Int J Inf Manag. 2015;35(2):137-44.

53. Callaghan CT, Rowley JJL, Cornwell WK, Poore AGB, Major RE. Improving big citizen science data: Moving beyond haphazard sampling. PLoS Biol. 2019;17(6):e3000357.

54. Welvaert M, Caley P. Citizen surveillance for environmental monitoring: combining the efforts of citizen science and crowdsourcing in a quantitative data framework. Springerplus. 2016;5(1):1890.

55. Cove MV, Kays R, Bontrager H, Bresnan C, Lasky M, Frerichs $T$, et al. SNAPSHOT USA 2019: a coordinated national camera trap survey of the United States. Ecology. n/a(n/a):e03353.

56. Rowley JJL, Callaghan CT, Cutajar T. FrogID: Citlzen scientists provide validated biodiversity data on frogs of Australla. Herpetol Conserv Biol. 2019;14:155-70.

57. Holyoak M, Casagrandi R, Nathan R, Revilla E, Spiegel O. Trends and missing parts in the study of movement ecology. Proc Natl Acad Sci. 2008;105(49):19060-5.

58. Kelling S, Johnston A, Bonn A, Fink D, Ruiz-Gutierrez V, Bonney R, et al. Using semistructured surveys to improve citizen science data for monitoring biodiversity. Bioscience. 2019;69(3):170-9.

59. Fox R, Warren MS, Brereton TM, Roy DB, Robinson A. A new Red List of British butterflies. Insect Conserv Divers. 2011;4(3):159-72.

60. Huuskonen A, Saltikoff E, Holleman I. The operational weather radar network in Europe. Bull Am Meteorol Soc. 2014;95(6):897-907.

61. Van Horn G, Mac Aodha O, Song Y, Cui Y, Sun C, Shepard A, et al. The iNaturalist species classification and detection dataset. In: 2018 IEEE/ CVF conference on computer vision and pattern recognition. Salt Lake City, UT: IEEE; 2018 [cited 2021 May 27]. p. 8769-78. https:// ieeexplore.ieee.org/document/8579012/.

62. Prudic KL, McFarland KP, Oliver JC, Hutchinson RA, Long EC, Kerr JT, et al. eButterfly: leveraging massive online citizen science for butterfly conservation. Insects. 2017;8(2):53.

63. Bonney R, Cooper CB, Dickinson J, Kelling S, Phillips T, Rosenberg $\mathrm{KV}$, et al. Citizen science: a developing tool for expanding science knowledge and scientific literacy. Bioscience. 2009;59(11):977-84.

64. Planillo A, Fiechter L, Sturm U, Voigt-Heucke S, Kramer-Schadt S. Citizen science data for urban planning: comparing different sampling schemes for modelling urban bird distribution. Landsc Urban Plan. 2021:5:211.

65. Rapacciuolo G, Young A, Johnson R. Deriving indicators of biodiversity change from unstructured community-contributed data. Oikos. 2021. https://doi.org/10.1111/oik.08215.

66. Johnston A, Hochachka WM, Strimas-Mackey ME, Gutierrez VR, Robinson OJ, Miller ET, et al. Analytical guidelines to increase the value of community science data: an example using eBird data to estimate species distributions. Divers Distrib. 2021. https://doi.org/10.1111/ddi. 13271.

67. Fink D, Hochachka WM, Zuckerberg B, Winkler DW, Shaby B, Munson MA, et al. Spatiotemporal exploratory models for broad-scale survey data. Ecol Appl Publ Ecol Soc Am. 2010;20(8):2131-47.

68. Sicacha-Parada J, Steinsland I, Cretois B, Borgelt J. Accounting for spatial varying sampling effort due to accessibility in Citizen Science data: a case study of moose in Norway. Spat Stat. 2021;42:100446.

69. Ditmer MA, lannarilli F, Tri AN, Garshelis DL, Carter NH. Artificial night light helps account for observer bias in citizen science monitoring of an expanding large mammal population. J Anim Ecol. 2021;90(2):330-42.

70. GBIF. What is GBIF? [Internet]. The Global Biodiversity Information Facility. 2021 [cited 2021 May 20]. https://www.gbif.org/

71. HerpMapper. HerpMapper - Global Herp Atlas [Internet]. HerpMapper. [cited 2021 May 20]. https://www.herpmapper.org/

72. Pollard E. A method for assessing changes in the abundance of butterflies. Biol Conserv. 1977;12(2):115-34.

73. Pollard E, Yates TJ. Monitoring butterflies for ecology and conservation: the British butterfly monitoring scheme. Berlin: Springer; 1994. p. 296.

74. NEXRAD on AWS - Registry of Open Data on AWS [Internet]. 2021 [cited 2021 May 20]. https://registry.opendata.aws/noaa-nexrad/.

75. Information (NCEI) NC for E, Information (NCEI) NC for E. NOAA Next Generation Radar (NEXRAD) Level 2 Base Data [Internet]. [cited 2021 May 20]. https://www.ncei.noaa.gov/access/metadata/landing-page/ bin/iso?id=gov.noaa.ncdc:C00345.

76. Holleman I, Delobbe L, Zgonc A. Update on the European weather radar network (OPERA). In: OPERA, Proceedings of ERAD 2008. 2008

77. McShea WJ, Forrester T, Costello R, He Z, Kays R. Volunteer-run cameras as distributed sensors for macrosystem mammal research. Landsc Ecol. 2016:31(1):55-66.

78. Rowley JJL, Callaghan CT. The FrogID dataset: expert-validated occurrence records of Australia's frogs collected by citizen scientists. ZooKeys. 2020;17(912):139-51.

79. Chilson PB, Bridge E, Frick WF, Chapman JW, Kelly JF. Radar aeroecology: exploring the movements of aerial fauna through radio-wave remote sensing. Biol Lett. 2012;8(5):698-701.

80. Shamoun-Baranes J, Bauer S, Chapman JW, Desmet P, Dokter AM, Farnsworth A, et al. Weather radars' role in biodiversity monitoring. Science. 2021; 372(6539). https://research.wur.nl/en/publications/weath er-radars-role-in-biodiversity-monitoring.

81. Chapman JW, Drake VA, Reynolds DR. Recent insights from radar studies of insect flight. Annu Rev Entomol. 2010;56(1):337-56.

82. Mizrahi D, Fogg T, Magarian V, Elia P, Hodgetts D, La Puma D. Radar monitoring of bird and bat movement patterns on block island and its coastal waters. 2010.

83. Heist KW, Bowden TS, Ferguson J, Rathbun NA, Olson EC, Nolfi DC, et al. Radar quantifies migrant concentration and Dawn reorientation at a Great Lakes shoreline. Mov Ecol. 2018;6(1):15.

84. Kelly JF, Pletschet SM. Accuracy of swallow roost locations assigned using weather surveillance radar. Remote Sens Ecol Conserv. 2018;4(2):166-72.

85. Bridge ES, Pletschet SM, Fagin T, Chilson PB, Horton KG, Broadfoot KR, et al. Persistence and habitat associations of Purple Martin roosts quantified via weather surveillance radar. Landsc Ecol. 2016;31(1):43-53.

86. Buler JJ, Lakshmanan V, La Puma D. Improving weather radar data processing for biological research applications: final report. 2012;15.

87. Dokter AM, Liechti F, Stark H, Delobbe L, Tabary P, Holleman I. Bird migration flight altitudes studied by a network of operational weather radars. J R Soc Interface. 2011;8(54):30-43.

88. Farnsworth A, Van Doren BM, Hochachka WM, Sheldon D, Winner $\mathrm{K}$, Irvine J, et al. A characterization of autumn nocturnal migration detected by weather surveillance radars in the northeastern USA. Ecol Appl Publ Ecol Soc Am. 2016;26(3):752-70. 
89. Lin T-Y, Winner K, Bernstein G, Mittal A, Dokter AM, Horton KG, et al. MistNet: measuring historical bird migration in the US using archived weather radar data and convolutional neural networks. Methods Ecol Evol. 2019;10(11):1908-22.

90. Sheldon D, Winner K, Bhambhani P, Bernstein G. darkecology/wsrlib: Version 0.2.0 [Internet]. Zenodo; 2019 [cited 2021 May 20]. https:// zenodo.org/record/3352264\#.YKavvoNKhH4.

91. Saltikoff E, Friedrich K, Soderholm J, Lengfeld K, Nelson B, Becker A, et al. An overview of using weather radar for climatological studies: successes, challenges, and potential. Bull Am Meteorol Soc. 2019;100(9):1739-52.

92. Nussbaumer R, Benoit L, Mariethoz G, Liechti F, Bauer S, Schmid B. Modelling the flow of nocturnal bird migration with year-round European weather radar network. bioRxiv. 2020;19.

93. Smith AD, Paton PWC, McWilliams SR. Using nocturnal flight calls to assess the fall migration of warblers and sparrows along a coastal ecological barrier. PLoS ONE. 2014;9(3):e92218.

94. Townsend PA, Clare J, Liu N, Stenglein JL, Anhalt-Depies C, Deelen $T R V$, et al. Integrating remote sensing and jurisdictional observation networks to improve the resolution of ecological management. bioRxiv. 2020;2020.06.08.140848.

95. Lasky M, Parsons A, Schuttler S, Mash A, Larson L, Norton B, et al. Candid critters: challenges and solutions in a large-scale citizen science camera trap project. Citiz Sci Theory Pract. 2021;6(1):4.

96. Banner KM, Irvine KM, Rodhouse TJ, Wright WJ, Rodriguez RM, Litt AR. Improving geographically extensive acoustic survey designs for modeling species occurrence with imperfect detection and misidentification. Ecol Evol. 2018;8(12):6144-56.

97. Chen G, Han TX, He Z, Kays R, Forrester T. Deep convolutional neural network based species recognition for wild animal monitoring. In: 2014 IEEE international conference on image processing (ICIP). 2014. p. 858-62.

98. Swanson A, Kosmala M, Lintott C, Simpson R, Smith A, Packer C. Snapshot Serengeti, high-frequency annotated camera trap images of 40 mammalian species in an African savanna. Sci Data. 2015;2(1):150026.

99. Glover-Kapfer P, Soto-Navarro CA, Wearn OR. Camera-trapping version 3.0: current constraints and future priorities for development. Remote Sens Ecol Conserv. 2019;5(3):209-23.

100. Wearn OR, Glover-Kapfer P. Snap happy: camera traps are an effective sampling tool when compared with alternative methods. R Soc Open Sci. 2019;6(3):181748.

101. Whytock RC, Świeżewski J, Zwerts JA, Bara-Słupski T, Pambo AFK, Rogala $M$, et al. Robust ecological analysis of camera trap data labelled by a machine learning model. Methods Ecol Evol. 2021. https://doi.org/10. 1111/2041-210X.13576.

102. Sugai LSM, Desjonquères $C$, Silva TSF, Llusia D. A roadmap for survey designs in terrestrial acoustic monitoring. Remote Sens Ecol Conserv. 2020;6(3):220-35

103. lannarilli F, Erb J, Arnold TW, Fieberg JR. Evaluating species-specific responses to camera-trap survey designs. Wildl Biol. 2021. https://doi. org/10.2981/wlb.00726.full.

104. Reichert BE, Bayless M, Cheng TL, Coleman JTH, Francis CM, Frick WF, et al. NABat: a top-down, bottom-up solution to collaborative continental-scale monitoring. Ambio. 2021;50(4):901-13.

105. Tape KD, Gustine DD. Capturing migration phenology of terrestrial wildlife using camera traps. Bioscience. 2014;64(2):117-24.

106. Farnsworth A. Flight calls and their value for future ornithological studies and conservation research. Auk. 2005;122(3):733-46.

107. Watson MJ, Wilson DR, Mennill DJ. Anthropogenic light is associated with increased vocal activity by nocturnally migrating birds. The Condor. 2016;118(2):338-44

108. Gibb R, Browning E, Glover-Kapfer P, Jones KE. Emerging opportunities and challenges for passive acoustics in ecological assessment and monitoring. Methods Ecol Evol. 2019:10(2):169-85.

109. Mackenzie DI, Nichols JD, Royle JA, Pollock KH, Bailey L, Hines JE. Occupancy estimation and modeling: inferring patterns and dynamics of species occurrence. Amsterdam: Elsevier; 2017. p. 668.

110. Redhead JW, Fox R, Brereton T, Oliver TH. Assessing species' habitat associations from occurrence records, standardised monitoring data and expert opinion: a test with British butterflies. Ecol Indic. 2016:1(62):271-8
111. Pocock MJO, Tweddle JC, Savage J, Robinson LD, Roy HE. The diversity and evolution of ecological and environmental citizen science. PLoS ONE. 2017;12(4):e0172579.

112. Farley SS, Dawson A, Goring SJ, Williams JW. Situating ecology as a bigdata science: current advances, challenges, and solutions. Bioscience. 2018;68(8):563-76.

113. Gauthreaux SA, Belser CG. Displays of bird movements on the WSR-88D: patterns and quantification. Weather Forecast. 1998;13(2):453-64.

114. Gauthreaux SA Jr, Livingston JW, Belser CG. Detection and discrimination of fauna in the aerosphere using Doppler weather surveillance radar. Integr Comp Biol. 2008;48(1):12-23.

115. Buler J, DiehI R. Quantifying bird density during migratory stopover using weather surveillance radar. IEEE Trans Geosci Remote Sens. 2009;47(8):2741-51.

116. WDSS-II [Internet]. [cited 2021 May 20]. http://www.wdssii.org/.

117. Dokter AM, Desmet P, Spaaks JH, van Hoey S, Veen L, Verlinden L, et al. bioRad: biological analysis and visualization of weather radar data. Ecography. 2019;42(5):852-60.

118. Norouzzadeh MS, Nguyen A, Kosmala M, Swanson A, Palmer MS, Packer C, et al. Automatically identifying, counting, and describing wild animals in camera-trap images with deep learning. Proc Natl Acad Sci. 2018;115(25):E5716-25.

119. Schneider S, Taylor GW, Kremer SC. Deep Learning Object Detection Methods for Ecological Camera Trap Data. ArXiv180310842 Cs [Internet]. 2018 Mar 28 [cited 2021 May 20]. http://arxiv.org/abs/1803.10842.

120. Tabak MA, Norouzzadeh MS, Wolfson DW, Sweeney SJ, Vercauteren KC, Snow NP, et al. Machine learning to classify animal species in camera trap images: applications in ecology. Methods Ecol Evol. 2019;10(4):585-90.

121. Willi M, Pitman RT, Cardoso AW, Locke C, Swanson A, Boyer A, et al. Identifying animal species in camera trap images using deep learning and citizen science. Methods Ecol Evol. 2019;10(1):80-91.

122. Weinstein BG. MotionMeerkat: integrating motion video detection and ecological monitoring. Methods Ecol Evol. 2015;6(3):357-62.

123. Zamora-Gutierrez V, Ortega J, Avila-Flores R, Aguilar-Rodríguez PA, Alarcón-Montano M, Avila-Torresagatón LG, et al. The Sonozotz project: assembling an echolocation call library for bats in a megadiverse country. Ecol Evol. 2020;10(11):4928-43.

124. Fink D, Auer T, Johnston A, Ruiz-Gutierrez V, Hochachka WM, Kelling S. Modeling avian full annual cycle distribution and population trends with citizen science data. Ecol Appl. 2020;30(3):e02056.

125. Greig El, Wood EM, Bonter DN. Winter range expansion of a hummingbird is associated with urbanization and supplementary feeding. Proc $R$ Soc B Biol Sci. 1852;2017(284):20170256.

126. Bonter DN, Cooper CB. Data validation in citizen science: a case study from Project FeederWatch. Front Ecol Environ. 2012;10(6):305-7.

127. Reichert BE, Lausen C, Loeb S, Weller T, Allen R, Britzke E, et al. A guide to processing bat acoustic data for the North American Bat Monitoring Program (NABat). U.S. Geological Survey; 2018 p. 33 p. (Open-File Report). Report No.: 2018-1068.

128. Knape J, Korner-Nievergelt F. On assumptions behind estimates of abundance from counts at multiple sites. Methods Ecol Evol. 2016;7(2):206-9.

129. Link WA, Schofield MR, Barker RJ, Sauer JR. On the robustness of N-mixture models. Ecology. 2018;99(7):1547-51.

130. O'Connell AF, Bailey LL. Inference for Occupancy and Occupancy Dynamics. In: O'Connell AF, Nichols JD, Karanth KU, editors. Camera Traps in Animal Ecology: Methods and Analyses [Internet]. Tokyo: Springer Japan; 2011 [cited 2021 May 20]. p. 191-204. https://doi.org/ 10.1007/978-4-431-99495-4_11.

131. Miller DAW, Pacifici K, Sanderlin JS, Reich BJ. The recent past and promising future for data integration methods to estimate species' distributions. Methods Ecol Evol. 2019;10(1):22-37.

132. Fletcher RJ, Hefley TJ, Robertson EP, Zuckerberg B, McCleery RA, Dorazio RM. A practical guide for combining data to model species distributions. Ecology. 2019;100(6):e02710.

133. Isaac NJB, Jarzyna MA, Keil P, Dambly LI, Boersch-Supan PH, Browning $E$, et al. Data integration for large-scale models of species distributions. Trends Ecol Evol. 2020;35(1):56-67. 
134. Supp SR, La Sorte FA, Cormier TA, Lim MCW, Powers DR, Wethington SM, et al. Citizen-science data provides new insight into annual and seasonal variation in migration patterns. Ecosphere. 2015;6(1):art15.

135. Johnston A, Moran N, Musgrove A, Fink D, Baillie SR. Estimating species distributions from spatially biased citizen science data. Ecol Model. 2020;422:108927.

136. Scotson L, Fredriksson G, Ngoprasert D, Wong W-M, Fieberg J. Projecting range-wide sun bear population trends using tree cover and camera-trap bycatch data. PLoS ONE. 2017;12(9):e0185336.

137. Warton DI, Renner IW, Ramp D. Model-based control of observer bias for the analysis of presence-only data in ecology. PLoS ONE. 2013;8(11):e79168.

138. Fithian W, Elith J, Hastie T, Keith DA. Bias correction in species distribution models: pooling survey and collection data for multiple species. Methods Ecol Evol. 2015;6(4):424-38.

139. Phillips SJ, Dudík M, Elith J, Graham CH, Lehmann A, Leathwick J, et al. Sample selection bias and presence-only distribution models: implications for background and pseudo-absence data. Ecol Appl Publ Ecol Soc Am. 2009;19(1):181-97.

140. Botella C, Joly A, Monestiez P, Bonnet P, Munoz F. Bias in presence-only niche models related to sampling effort and species niches: lessons for background point selection. PLOS ONE. 2020;15(5):e0232078.

141. Pacifici K, Reich BJ, Miller DAW, Gardner B, Stauffer G, Singh S, et al. Integrating multiple data sources in species distribution modeling: a framework for data fusion*. Ecology. 2017;98(3):840-50.

142. Matthiopoulos J, Fieberg J, Aarts G. Species-Habitat Associations: Spatial data, predictive models, and ecological insights [Internet]. University of Minnesota Libraries Publishing; 2020 [cited 2021 May 27]. http://conse rvancy.umn.edu/handle/11299/217469.

143. Fan J, Han F, Liu H. Challenges of big data analysis. Natl Sci Rev. 2014;1(2):293-314.

144. Dormann CF. Effects of incorporating spatial autocorrelation into the analysis of species distribution data. Glob Ecol Biogeogr. 2007;16(2):129-38.

145. Record S, Fitzpatrick MC, Finley AO, Veloz S, Ellison AM. Should species distribution models account for spatial autocorrelation? A test of model projections across eight millennia of climate change. Glob Ecol Biogeogr. 2013;22(6):760-71.

146. Miller JA. Species distribution models: spatial autocorrelation and nonstationarity. Prog Phys Geogr Earth Environ. 2012;36(5):681-92.

147. Roberts DR, Bahn V, Ciuti S, Boyce MS, Elith J, Guillera-Arroita G, et al. Cross-validation strategies for data with temporal, spatial, hierarchical, or phylogenetic structure. Ecography. 2017;40(8):913-29.

148. Schliep EM, Lany NK, Zarnetske PL, Schaeffer RN, Orians CM, Orwig DA, et al. Joint species distribution modelling for spatio-temporal occurrence and ordinal abundance data. Glob Ecol Biogeogr. 2018:27(1):142-55.

149. De Marco PJ, Nóbrega CC. Evaluating collinearity effects on species distribution models: an approach based on virtual species simulation. PLOS ONE. 2018;13(9):e0202403.

150. Dormann CF, Elith J, Bacher S, Buchmann C, Carl G, Carré G, et al. Collinearity: a review of methods to deal with it and a simulation study evaluating their performance. Ecography. 2013;36(1):27-46.

151. Fourcade $Y$, Besnard AG, Secondi J. Paintings predict the distribution of species, or the challenge of selecting environmental predictors and evaluation statistics. Glob Ecol Biogeogr. 2018;27(2):245-56.

152. Nilsson C, Dokter AM, Verlinden L, Shamoun-Baranes J, Schmid B, Desmet $P$, et al. Revealing patterns of nocturnal migration using the European weather radar network. Ecography. 2019;42(5):876-86.

153. La Sorte FA, Fink D. Migration distance, ecological barriers and en-route variation in the migratory behaviour of terrestrial bird populations. Glob Ecol Biogeogr. 2017;26(2):216-27.

154. Coleman T, Mentch L, Fink D, La Sorte FA, Winkler DW, Hooker G, et al. Statistical inference on tree swallow migrations with random forests. J $\mathrm{R}$ Stat Soc Ser C Appl Stat. 2020;69(4):973-89.

155. Hurlbert AH, Liang Z. Spatiotemporal variation in avian migration phenology: citizen science reveals effects of climate change. PLOS ONE. 2012;7(2):e31662

156. Wood SN. Generalized Additive Models: An Introduction with R, Second Edition [Internet]. Routledge \& CRC Press. 2006 [cited 2021 May 28].
https://www.routledge.com/Generalized-Additive-Models-An-Intro duction-with-R-Second-Edition/Wood/p/book/9781498728331.

157. Fink D, Damoulas T, Dave J. Adaptive spatio-temporal exploratory models: hemisphere-wide species distributions from massively crowdsourced eBird data. Proc AAAI Conf Artif Intell. 2013;27(1). https://ojs. aaai.org/index.php/AAAl/article/view/8484.

158. Wikle CK. Hierarchical Bayesian models for predicting the spread of ecological processes. Ecology. 2003;84(6):1382-94.

159. Auger-Méthé $M$, Newman $K$, Cole D, Empacher F, Gryba R, King AA, et al. A guide to state-space modeling of ecological time series. Ecol Monogr. in press;n/a(n/a):e01470.

160. Wilman H, Belmaker J, Simpson J, de la Rosa C, Rivadeneira MM, Jetz W. EltonTraits 1.0: species-level foraging attributes of the world's birds and mammals. Ecology. 2014;95(7):2027-2027.

161. Sheard C, Neate-Clegg MHC, Alioravainen N, Jones SEl, Vincent C, MacGregor HEA, et al. Ecological drivers of global gradients in avian dispersal inferred from wing morphology. Nat Commun. 2020;11(1):2463.

162. Jeliazkov A, Mijatovic D, Chantepie S, Andrew N, Arlettaz R, Barbaro L, et al. A global database for metacommunity ecology, integrating species, traits, environment and space. Sci Data. 2020;7(1):6.

163. Bolker BM, Brooks ME, Clark CJ, Geange SW, Poulsen JR, Stevens MHH, et al. Generalized linear mixed models: a practical guide for ecology and evolution. Trends Ecol Evol. 2009:24(3):127-35.

164. Pinheiro J, Bates D. Mixed-effects models in S and S-PLUS. New York: Springer; 2000. (Statistics and Computing). https://www.springer.com/ gp/book/9780387989570.

165. La Sorte FA, Fink D, Hochachka WM, Kelling S. Convergence of broadscale migration strategies in terrestrial birds. Proc R Soc B Biol Sci. 1823;2016(283):20152588.

166. Menz MHM, Brown BV, Wotton KR. Quantification of migrant hoverfly movements (Diptera: Syrphidae) on the West Coast of North America. R Soc Open Sci. 2019;6(4):190153.

167. Mayor SJ, Guralnick RP, Tingley MW, Otegui J, Withey JC, Elmendorf SC, et al. Increasing phenological asynchrony between spring green-up and arrival of migratory birds. Sci Rep. 2017:7(1):1902.

168. Sivakumar AH, Sheldon D, Winner K, Burt CS, Horton KG. A weather surveillance radar view of Alaskan avian migration. Proc R Soc B Biol Sci. 1950:2021(288):20210232

169. Hooten MB, Wikle CK. A hierarchical Bayesian non-linear spatio-temporal model for the spread of invasive species with application to the Eurasian Collared-Dove. Environ Ecol Stat. 2008;15(1):59-70.

170. Williams JE, Blois JL. Range shifts in response to past and future climate change: can climate velocities and species' dispersal capabilities explain variation in mammalian range shifts? J Biogeogr. 2018;45(9):2175-89.

171. Youngflesh C, Socolar J, Amaral BR, Arab A, Guralnick RP, Hurlbert AH, et al. Migratory strategy drives species-level variation in bird sensitivity to vegetation green-up. Nat Ecol Evol. 2021;29:1-8.

172. La Sorte FA, Fink D, Hochachka WM, Farnsworth A, Rodewald AD, Rosenberg KV, et al. The role of atmospheric conditions in the seasonal dynamics of North American migration flyways. J Biogeogr. 2014:41(9):1685-96.

173. La Sorte FA, Fink D, Hochachka WM, DeLong JP, Kelling S. Spring phenology of ecological productivity contributes to the use of looped migration strategies by birds. Proc R Soc B Biol Sci. 2014;281(1793):20140984.

174. Flockhart DTT, Larrivée M, Prudic KL, Norris DR. Estimating the annual distribution of monarch butterflies in Canada over 16 years using citizen science data. FACETS. 2019. https://doi.org/10.1139/ facets-2018-0011.

175. La Sorte FA, Hochachka WM, Farnsworth A, Sheldon D, Fink D, Geevarghese J, et al. Migration timing and its determinants for nocturnal migratory birds during autumn migration. J Anim Ecol. 2015;84(5):1202-12

176. Zuckerberg B, Fink D, La Sorte FA, Hochachka WM, Kelling S. Nove seasonal land cover associations for eastern North American forest birds identified through dynamic species distribution modelling. Divers Distrib. 2016;22(6):717-30

177. La Sorte FA, Fink D, Blancher PJ, Rodewald AD, Ruiz-Gutierrez V, Rosenberg KV, et al. Global change and the distributional dynamics of migratory bird populations wintering in Central America. Glob Change Biol. 2017:23(12):5284-96. 
178. Pettorelli N, Smith J, Pecl GT, Hill JK, Norris K. Anticipating arrival: tackling the national challenges associated with the redistribution of biodiversity driven by climate change. J Appl Ecol. 2019;56(10):2298-304.

179. La Sorte FA, Fink D, Buler JJ, Farnsworth A, Cabrera-Cruz SA. Seasonal associations with urban light pollution for nocturnally migrating bird populations. Glob Change Biol. 2017;23(11):4609-19.

180. La Sorte FA, Horton KG. Seasonal variation in the effects of artificial light at night on the occurrence of nocturnally migrating birds in urban areas. Environ Pollut. 2021;270:116085.

181. Vardi R, Berger-Tal O, Roll U. iNaturalist insights illuminate COVID19 effects on large mammals in urban centers. Biol Conserv. 2021;254:108953.

182. Johnston A, Auer T, Fink D, Strimas-Mackey M, lliff M, Rosenberg $\mathrm{KV}$, et al. Comparing abundance distributions and range maps in spatial conservation planning for migratory species. Ecol Appl. 2020;30(3):e02058.

183. Flockhart DTT, Wassenaar LI, Martin TG, Hobson KA, Wunder MB, Norris DR. Tracking multi-generational colonization of the breeding grounds by monarch butterflies in eastern North America. Proc R Soc B Biol Sci. 2013:280(1768):20131087.

184. Linden DW, Sirén APK, Pekins PJ. Integrating telemetry data into spatial capture-recapture modifies inferences on multi-scale resource selection. Ecosphere. 2018;9(4):e02203.

185. Michelot T, Blackwell PG, Matthiopoulos J. Linking resource selection and step selection models for habitat preferences in animals. Ecology. 2019;100(1):e02452.

186. Lopez-Marcano S, Jinks EL, Buelow CA, Brown CJ, Wang D, Kusy B, et al. Automatic detection of fish and tracking of movement for ecology. Ecol Evol. 2021. https://doi.org/10.1002/ece3.7656.

187. Risch D, Castellote M, Clark CW, Davis GE, Dugan PJ, Hodge LE, et al. Seasonal migrations of North Atlantic minke whales: novel insights from large-scale passive acoustic monitoring networks. Mov Ecol. 2014;2(1):24.

188. Horvitz N, Wang R, Wan F-H, Nathan R. Pervasive human-mediated large-scale invasion: analysis of spread patterns and their underlying mechanisms in 17 of China's worst invasive plants. J Ecol. 2017;105(1):85-94.

189. Koide D, Yoshida K, Daehler CC, Mueller-Dombois D. An upward elevation shift of native and non-native vascular plants over 40 years on the island of Hawai'i. J Veg Sci. 2017;28(5):939-50.

190. Fan L, Chen S, Liang S, Sun X, Chen H, You L, et al. Assessing longterm spatial movement of wheat area across China. Agric Syst. 2020;185:102933.

\section{Publisher's Note}

Springer Nature remains neutral with regard to jurisdictional claims in published maps and institutional affiliations.

Ready to submit your research? Choose BMC and benefit from:

- fast, convenient online submission

- thorough peer review by experienced researchers in your field

- rapid publication on acceptance

- support for research data, including large and complex data types

- gold Open Access which fosters wider collaboration and increased citations

- maximum visibility for your research: over 100M website views per year

At BMC, research is always in progress.

Learn more biomedcentral.com/submissions 